\title{
$q$-SELBERG INTEGRALS AND MACDONALD POLYNOMIALS
}

\author{
S. OLE WARNAAR
}

Dedicated to Richard Askey on the occasion of his 70th birthday

\begin{abstract}
Using the theory of Macdonald polynomials, a number of $q$-integrals of Selberg type are proved.
\end{abstract}

\section{INTRODUCTION AND SUMMARY}

In [3, Richard Askey conjectured several $q$-integrals extending the famous Selberg integral. The third of his now ex-conjectures reads

$$
\begin{aligned}
\int_{[0, \infty)^{n}} & \prod_{i=1}^{n} x_{i}^{\alpha-1} \frac{\left(-c x_{i} q^{\alpha+\beta+2(n-1) k} ; q\right)_{\infty}}{\left(-c x_{i} ; q\right)_{\infty}} \prod_{1 \leq i<j \leq n} x_{i}^{2 k}\left(\frac{x_{j} q^{1-k}}{x_{i}} ; q\right)_{2 k} \mathrm{~d}_{q} x \\
= & \prod_{i=1}^{n} \frac{\Gamma_{q}(\alpha+(i-1) k) \Gamma_{q}(\beta+(i-1) k) \Gamma_{q}(i k+1)}{\Gamma_{q}(\alpha+\beta+(n+i-2) k) \Gamma_{q}(k+1)} \frac{\vartheta\left(-c q^{\alpha+2(i-1) k} ; q\right)}{\vartheta(-c ; q)} .
\end{aligned}
$$

with $k$ a positive integer, $\operatorname{Re}(\alpha)>0, \operatorname{Re}(\beta)>0$ and $0<q<1$. Here $(a ; q)_{m}=$ $\prod_{j=0}^{m-1}\left(1-a q^{j}\right)$ and $(a ; q)_{\infty}=\prod_{j=0}^{\infty}\left(1-a q^{j}\right)$ are $q$-shifted factorials,

$$
\Gamma_{q}(z)=(1-q)^{1-z} \frac{(q ; q)_{\infty}}{\left(q^{z} ; q\right)_{\infty}}
$$

is the $q$-gamma function,

$$
\vartheta(z ; q)=(z ; q)_{\infty}(q / z ; q)_{\infty}
$$

is a theta function, and, for $x=\left(x_{1}, \ldots, x_{n}\right)$ and measure $\mathrm{d}_{q} x=\mathrm{d}_{q} x_{1} \ldots \mathrm{d}_{q} x_{n}$,

$$
\int_{[0, \infty)^{n}} f(x) \mathrm{d}_{q} x=(1-q)^{n} \sum_{k_{1}, \ldots, k_{n}=-\infty}^{\infty} f\left(q^{k_{1}}, \ldots, q^{k_{n}}\right) q^{k_{1}+\cdots+k_{n}}
$$

is a multiple $q$-integral.

To see that (1.1) generalizes a special case of the Selberg integral 24]

$$
\begin{aligned}
\int_{[0,1]^{n}} \prod_{i=1}^{n} x_{i}^{\alpha-1}\left(1-x_{i}\right)^{\beta-1} & \prod_{1 \leq i<j \leq n}\left|x_{i}-x_{j}\right|^{2 \gamma} \mathrm{d} x \\
& =\prod_{i=1}^{n} \frac{\Gamma(\alpha+(i-1) \gamma) \Gamma(\beta+(i-1) \gamma) \Gamma(i \gamma+1)}{\Gamma(\alpha+\beta+(n+i-2) \gamma) \Gamma(\gamma+1)}
\end{aligned}
$$

2000 Mathematics Subject Classification. 33D05, 33D52, 33D60. 
true for $\operatorname{Re}(\alpha)>0, \operatorname{Re}(\beta)>0$ and $\operatorname{Re}(\gamma)>-\min \{1 / n, \operatorname{Re}(\alpha) /(n-1), \operatorname{Re}(\beta) /(n-$ $1)\}$, assume that $c>0$ and let $q$ tend to one in 1.1 using the formal limits

$$
\lim _{q \rightarrow 1}(a ; q)_{\infty} /\left(a q^{z} ; q\right)_{\infty}=(1-a)^{z},
$$

$\lim _{q \rightarrow 1} \Gamma_{q}(z)=\Gamma(z)$ and

$$
\lim _{q \rightarrow 1} \int_{[0, \infty)^{n}} f(x) \mathrm{d}_{q} x=\int_{[0, \infty)^{n}} f(x) \mathrm{d} x
$$

with $\mathrm{d} x=\mathrm{d} x_{1} \ldots \mathrm{d} x_{n}$. After rescaling $c x_{i} \mapsto x_{i}$ this yields the integral 1, Exercise $8.14 ; \gamma=k]$

$$
\begin{aligned}
& \int_{[0, \infty)^{n}} \prod_{i=1}^{n} \frac{x_{i}^{\alpha-1}}{\left(1+x_{i}\right)^{\alpha+\beta+2(n-1) k}} \prod_{1 \leq i<j \leq n}\left(x_{i}-x_{j}\right)^{2 k} \mathrm{~d} x \\
&=\prod_{i=1}^{n} \frac{\Gamma(\alpha+(i-1) k) \Gamma(\beta+(i-1) k) \Gamma(i k+1)}{\Gamma(\alpha+\beta+(n+i-2) k) \Gamma(k+1)},
\end{aligned}
$$

for $\operatorname{Re}(\alpha)>0$ and $\operatorname{Re}(\beta)>0$. By the variable change $x_{i} \mapsto x_{i} /\left(1-x_{i}\right)$ this becomes the Selberg integral (1.5) for $\gamma=k$.

Askey's conjecture (1.1) was proved for $k=1$ (and up to a symmetrization of the integrand) by Milne [22, Theorem 4.18] and by Aomoto [2] for general $k$. Subsequently Kaneko [18 found a proof based on a ${ }_{1} \Psi_{1}$ summation for Macdonald polynomials. We will show that Kaneko's ${ }_{1} \Psi_{1}$ sum together with a symmetry property of the Macdonald polynomials implies an integral evaluation more general than (1.1).

Theorem 1.1. Let $\lambda=\left(\lambda_{1}, \ldots, \lambda_{n}\right)$ be a partition and $k$ a positive integer. Then

$$
\begin{aligned}
\int_{[0, \infty)^{n}} P_{\lambda}\left(x ; q, q^{k}\right) \prod_{i=1}^{n} x_{i}^{\alpha-1} \frac{\left(-c x_{i} q^{\alpha+\beta+2(n-1) k} ; q\right)_{\infty}}{\left(-c x_{i} ; q\right)_{\infty}} \prod_{1 \leq i<j \leq n} x_{i}^{2 k}\left(\frac{x_{j} q^{1-k}}{x_{i}} ; q\right)_{2 k} \mathrm{~d}_{q} x \\
=P_{\lambda}\left(q^{k \delta} ; q, q^{k}\right) \prod_{i=1}^{n} q^{-k(2 n-3 i+1) \lambda_{i}} \frac{\vartheta\left(-c q^{\alpha+2(i-1) k+\lambda_{i}} ; q\right)}{\vartheta(-c ; q)} \\
\times \frac{\Gamma_{q}\left(\alpha+(n-i) k+\lambda_{i}\right) \Gamma_{q}\left(\beta+(i-1) k-\lambda_{i}\right) \Gamma_{q}(i k+1)}{\Gamma_{q}(\alpha+\beta+(n+i-2) k) \Gamma_{q}(k+1)} \\
=q^{\alpha k\left(\begin{array}{c}
n \\
2
\end{array}\right)+2 k^{2}\left(\begin{array}{c}
n \\
3
\end{array}\right)} P_{\lambda}\left(q^{k \delta} ; q, q^{k}\right) \prod_{i=1}^{n} \frac{\vartheta\left(-c q^{\alpha+(2 n-i-1) k+\lambda_{i}} ; q\right)}{\vartheta\left(-c q^{(n-i) k} ; q\right)_{\infty}} \\
\times \frac{\Gamma_{q}\left(\alpha+(n-i) k+\lambda_{i}\right) \Gamma_{q}\left(\beta+(i-1) k-\lambda_{i}\right) \Gamma_{q}(i k+1)}{\Gamma_{q}(\alpha+\beta+(n+i-2) k) \Gamma_{q}(k+1)}
\end{aligned}
$$

for $\operatorname{Re}(\alpha)>-\lambda_{n}, \operatorname{Re}(\beta)>\lambda_{1}$ and $0<q<1$.

Here $P_{\lambda}(x ; q, t)$ is a Macdonald polynomial (see Section 2 and $P_{\lambda}\left(q^{k \delta} ; q, q^{k}\right)$ denotes the specialized Macdonald polynomial $P_{\lambda}\left(1, q^{k}, \ldots, q^{(n-1) k} ; q, q^{k}\right)$ which can be expressed in closed form as

$$
P_{\lambda}\left(q^{k \delta} ; q, q^{k}\right)=q^{k n(\lambda)} \prod_{1 \leq i<j \leq n} \frac{\left(q^{(j-i) k+\lambda_{i}-\lambda_{j}} ; q\right)_{k}}{\left(q^{(j-i) k} ; q\right)_{k}}
$$


with $n(\lambda)=\sum_{i=1}^{n}(i-1) \lambda_{i}$.

The equality of the two expressions on the right of the theorem simply follows from the (multiplicative) quasi-periodicity of the theta functions:

$$
\vartheta\left(a q^{j} ; q\right)=(-a)^{-j} q^{-\left(\begin{array}{l}
j \\
2
\end{array}\right)} \vartheta(a ; q) .
$$

Denoting the empty partition by 0 , we have $P_{0}(x ; q, t)=1$. Hence the $\lambda=0$ case of the theorem reduces to (1.1). Also, since

$$
P_{\lambda}(x ; q, t)=\left(x_{1} \ldots x_{n}\right)^{\lambda_{n}} P_{\left(\lambda_{1}-\lambda_{n}, \lambda_{2}-\lambda_{n}, \ldots, 0\right)}(x ; q, t)
$$

it is easily seen by making the variable changes $\alpha \mapsto \alpha-\lambda_{n}$ and $\beta \mapsto \beta+\lambda_{n}$ that the $\lambda_{n}>0$ case of the theorem reduces to the $\lambda_{n}=0$ case. We also mention that Theorem 1.1 remains correct for $k=0$. However, here and elsewhere in the paper, we exclude $k=0$ for being trivial but requiring special attention. For example, for $k=0$ we have $P_{\lambda}(x ; q, 1)=m_{\lambda}(x)$ with $m_{\lambda}$ the monomial symmetric function, given by $m_{\lambda}(x)=\sum x_{1}^{\nu_{1}} \ldots x_{n}^{\nu_{n}}$ where the sum is over all distinct permutations $\nu$ of $\lambda$. Hence (1.7) for $k=0$ is incorrect unless $\lambda=(a, \ldots, a)=\left(a^{n}\right)$. However, with the correct interpretation of $P_{\lambda}(1, \ldots, 1 ; q, 0)=m_{\lambda}(1, \ldots, 1)$ the integral of Theorem 1.1 with $k=0$ trivializes to a sum over the one-dimensional integral (with $\alpha \mapsto \alpha+\mu_{i}$ and $\left.\beta \mapsto \beta-\mu_{i}\right)$

$$
\int_{0}^{\infty} x^{\alpha-1} \frac{\left(-c x q^{\alpha+\beta} ; q\right)_{\infty}}{(-c x ; q)_{\infty}} \mathrm{d}_{q} x=\frac{\Gamma_{q}(\alpha) \Gamma_{q}(\beta)}{\Gamma_{q}(\alpha+\beta)} \frac{\vartheta\left(-c q^{\alpha} ; q\right)}{\vartheta(-c ; q)} .
$$

This result, which is equivalent to Ramanujan's ${ }_{1} \psi_{1}$ summation [3, [5], corresponds to (1.1) with $n=1$.

A more interesting special case of the theorem is obtained in the $q \rightarrow 1$ limit for $c>0$. By rescaling $x$ to eliminate $c$ this yields the following generalization of the integration formula 1.6 .

Corollary 1.2. With the same conditions as in Theorem 1.1 there holds

$$
\begin{aligned}
& \int_{[0, \infty)^{n}} P_{\lambda}^{(1 / k)}(x) \prod_{i=1}^{n} \frac{x_{i}^{\alpha-1}}{\left(1+x_{i}\right)^{\alpha+\beta+2(n-1) k}} \prod_{1 \leq i<j \leq n}\left(x_{i}-x_{j}\right)^{2 k} \mathrm{~d} x \\
& \quad=P_{\lambda}^{(1 / k)}(1, \ldots, 1) \prod_{i=1}^{n} \frac{\Gamma\left(\alpha+(n-i) k+\lambda_{i}\right) \Gamma\left(\beta+(i-1) k-\lambda_{i}\right) \Gamma(i k+1)}{\Gamma(\alpha+\beta+(n+i-2) k) \Gamma(k+1)} .
\end{aligned}
$$

Here $P_{\lambda}^{(\alpha)}(x)$ is a Jack polynomial and

$$
P_{\lambda}^{(1 / k)}(1, \ldots, 1)=\prod_{1 \leq i<j \leq n} \frac{\left((j-i) k+\lambda_{i}-\lambda_{j}\right)_{k}}{((j-i) k)_{k}},
$$

with $(a)_{n}=\prod_{j=0}^{n-1}(a+j)$.

By replacing $q^{\beta} \mapsto-q^{1-\alpha-2(n-1) k} / c$ and then letting $c \mapsto-q^{\beta}$ in Theorem 1.1. and by noting that $\left(x_{i} q ; q\right)_{\infty}=0$ for $x_{i}=q^{k_{i}}$ with $k_{i}$ a negative integer, we obtain as second corollary another generalized $q$-Selberg integral. This integral was first conjectured by Kadell [11, Conjecture 8], and proved for $k=1$ by Kadell [11] and for general $k$ by Kaneko [16, Proposition 5.2] and Macdonald [20, Example VI.9.3]. See also Kadell [14, Theorem 1] for the $q \rightarrow 1$ limit. (To obtain the formulae of [11] and [20] one has to first carry out a symmetrization of the integrand.) 
Corollary 1.3. Let $\lambda$ be a partition and $k$ a positive integer. Then

$$
\begin{aligned}
& \int_{[0,1]^{n}} P_{\lambda}\left(x ; q, q^{k}\right) \prod_{i=1}^{n} x_{i}^{\alpha-1} \frac{\left(x_{i} q ; q\right)_{\infty}}{\left(x_{i} q^{\beta} ; q\right)_{\infty}} \prod_{1 \leq i<j \leq n} x_{i}^{2 k}\left(\frac{x_{j} q^{1-k}}{x_{i}} ; q\right)_{2 k} \mathrm{~d}_{q} x \\
&=q^{\alpha k\left(\begin{array}{c}
n \\
2
\end{array}\right)+2 k^{2}\left(\begin{array}{c}
n \\
3
\end{array}\right)} P_{\lambda}\left(q^{k \delta} ; q, q^{k}\right) \\
& \\
& \quad \times \prod_{i=1}^{n} \frac{\Gamma_{q}\left(\alpha+(n-i) k+\lambda_{i}\right) \Gamma_{q}(\beta+(i-1) k) \Gamma_{q}(i k+1)}{\Gamma_{q}\left(\alpha+\beta+(2 n-i-1) k+\lambda_{i}\right) \Gamma_{q}(k+1)}
\end{aligned}
$$

for $\operatorname{Re}(\alpha)>-\lambda_{n}, \operatorname{Re}(\beta)>0$ and $0<q<1$.

For $\lambda=0$ this is the well-known Askey-Habsieger-Kadell integral, conjectured by Askey [3, Conjecture 1] and proved independently by Habsieger [9] and Kadell [12, Theorem $2 ; l=m=0]$. In the above

$$
\int_{[0,1]^{n}} f(x) \mathrm{d}_{q} x=(1-q)^{n} \sum_{k_{1}, \ldots, k_{n}=0}^{\infty} f\left(q^{k_{1}}, \ldots, q^{k_{n}}\right) q^{k_{1}+\cdots+k_{n}} .
$$

The second main result of this paper entails a generalization of the $\beta=k$ case of Corollary 1.3

Theorem 1.4. For $\lambda=\left(\lambda_{1}, \ldots, \lambda_{n}\right)$ and $\mu=\left(\mu_{1}, \ldots, \mu_{n}\right)$ partitions and $k a$ positive integer there holds

$$
\begin{aligned}
& \int_{[0,1]^{n}} P_{\lambda}\left(x ; q, q^{k}\right) P_{\mu}\left(x ; q, q^{k}\right) \prod_{i=1}^{n} x_{i}^{\alpha-1}\left(x_{i} q ; q\right)_{k-1} \prod_{1 \leq i<j \leq n} x_{i}^{2 k}\left(\frac{x_{j} q^{1-k}}{x_{i}} ; q\right)_{2 k} \mathrm{~d}_{q} x \\
&=q^{\alpha k\left(\begin{array}{c}
n \\
2
\end{array}\right)+2 k^{2}\left(\begin{array}{l}
n \\
3
\end{array}\right)}(1-q)^{k n^{2}} P_{\lambda}\left(q^{k \delta} ; q, q^{k}\right) P_{\mu}\left(q^{k \delta} ; q, q^{k}\right) \\
& \times \prod_{i=1}^{n} \frac{\Gamma_{q}(i k) \Gamma_{q}(i k+1)}{\Gamma_{q}(k+1)} \prod_{i, j=1}^{n} \frac{1}{\left(q^{\alpha+(2 n-i-j) k+\lambda_{i}+\mu_{j}} ; q\right)_{k}},
\end{aligned}
$$

for $\operatorname{Re}(\alpha)>-\lambda_{n}-\mu_{n}$ and $0<q<1$.

In the limit when $q$ tends to 1 this reproduces the Hua-Kadell integration formula for Jack polynomials [10, Theorem 5.2.1], [13, Theorem 2].

Corollary 1.5. With the same conditions as in Theorem 1.4 there holds

$$
\begin{aligned}
\int_{[0,1]^{n}} P_{\lambda}^{(1 / k)}(x) P_{\mu}^{(1 / k)}(x) & \prod_{i=1}^{n} x_{i}^{\alpha-1}\left(1-x_{i}\right)^{k-1} \prod_{1 \leq i<j \leq n}\left(x_{i}-x_{j}\right)^{2 k} \mathrm{~d} x \\
& =n !(\Gamma(k))^{n} f_{\lambda}^{k} f_{\mu}^{k} \prod_{i, j=1}^{n} \frac{1}{\left(\alpha+(2 n-i-j) k+\lambda_{i}+\mu_{j}\right)_{k}},
\end{aligned}
$$

where

$$
f_{\lambda}^{k}=\prod_{1 \leq i<j \leq n}\left((j-i) k+\lambda_{i}-\lambda_{j}\right)_{k} .
$$

As another corollary of Theorem 1.4 we will prove the following integral representation of a terminating ${ }_{n+1} \Phi_{n}$ multivariable basic hypergeometric series based on Macdonald polynomials (see definition 2.16). 
Corollary 1.6. Let $y=\left(y_{1}, \ldots, y_{m}\right)$. For $\lambda=\left(\lambda_{1}, \ldots, \lambda_{n}\right)$ a partition and $k a$ positive integer there holds

$$
\begin{aligned}
& \int_{[0,1]^{n}} P_{\lambda}\left(x ; q, q^{k}\right) \prod_{\substack{1 \leq i \leq n \\
1 \leq j \leq m}}\left(1-x_{i} y_{j}\right) \\
& \times \prod_{i=1}^{n} x_{i}^{\alpha-1}\left(x_{i} q ; q\right)_{k-1} \prod_{1 \leq i<j \leq n} x_{i}^{2 k}\left(\frac{x_{j} q^{1-k}}{x_{i}} ; q\right)_{2 k} \mathrm{~d}_{q} x \\
&=q^{\alpha k\left(\begin{array}{l}
n \\
2
\end{array}\right)+2 k^{2}\left(\begin{array}{l}
n \\
3
\end{array}\right)} P_{\lambda}\left(q^{k \delta} ; q, q^{k}\right) \prod_{i=1}^{n} \frac{\Gamma_{q}\left(\alpha+(n-i) k+\lambda_{i}\right) \Gamma_{q}(i k) \Gamma_{q}(i k+1)}{\Gamma_{q}\left(\alpha+(2 n-i) k+\lambda_{i}\right) \Gamma_{q}(k+1)} \\
& \times{ }_{n+1} \Phi_{n}\left[\begin{array}{c}
q^{-n k} \\
q^{-\alpha-2(n-1) k-\lambda_{1}}, \ldots, q^{-\alpha-(n-1) k-\lambda_{n}}
\end{array} q^{k}, q, y\right] .
\end{aligned}
$$

for $\operatorname{Re}(\alpha)>-\lambda_{n}$ and $0<q<1$.

The generic numerator and denominator parameters in the above ${ }_{n+1} \Phi_{n}$ are $q^{-\alpha-(2 n-i-1) k-\lambda_{i}}$ and $q^{-\alpha-(2 n-i) k-\lambda_{i}}$. Equation $(1.10)$ extends the $\beta=k$ case of the following integral of Kaneko [17, Equation (3.1)]:

$$
\begin{gathered}
\int_{[0,1]^{n}} \prod_{\substack{1 \leq i \leq n \\
1 \leq j \leq m}}\left(1-x_{i} y_{j}\right) \prod_{i=1}^{n} x_{i}^{\alpha-1} \frac{\left(x_{i} q ; q\right)_{\infty}}{\left(x_{i} q^{\beta} ; q\right)_{\infty}} \prod_{1 \leq i<j \leq n} x_{i}^{2 k}\left(\frac{x_{j} q^{1-k}}{x_{i}} ; q\right)_{2 k} \mathrm{~d}_{q} x \\
=q^{\alpha k\left(\begin{array}{c}
n \\
2
\end{array}\right)+2 k^{2}\left(\begin{array}{c}
n \\
3
\end{array}\right)} \prod_{i=1}^{n} \frac{\Gamma_{q}(\alpha+(i-1) k) \Gamma_{q}(\beta+(i-1) k) \Gamma_{q}(i k+1)}{\Gamma_{q}(\alpha+\beta+(n+i-2) k) \Gamma_{q}(k+1)} \\
\times{ }_{2} \Phi_{1}\left[\begin{array}{c}
q^{-n k}, q^{-\alpha-(n-1) k} \\
q^{-\alpha-\beta-2(n-1) k}
\end{array} ; q^{k}, q, q^{k-\beta} y\right]
\end{gathered}
$$

true for $\operatorname{Re}(\alpha)>-\lambda_{n}, \operatorname{Re}(\beta)>0$ and $0<q<1$. Here $q^{k-\beta} y$ is shorthand for $\left(q^{k-\beta} y_{1}, \ldots, q^{k-\beta} y_{m}\right)$. When $m=1$ the above two ${ }_{r+1} \Phi_{r}$ series simplify to the ordinary one-variable basic hypergeometric series ${ }_{r+1} \phi_{r}$. We also note the remarkable fact that $m$-dimensional hypergeometric series are expressed in terms of $n$-dimensional $q$-integrals.

There is a well-known connection between constant term identities and $q$-Selberg integrals of the type given in Corollary 1.3 and Theorem 1.4 In the case of Corollary 1.3 the corresponding constant term identity was obtained by Kaneko. Correcting his expression for the exponent $D_{n}$ in [17, Equation (3.5)], Kaneko's identity asserts that [17, Theorem 4]

$$
\begin{aligned}
& \text { C.T. }\left[P_{\lambda}\left(x ; q, q^{k}\right) \prod_{i=1}^{n}\left(x_{i} ; q\right)_{a}\left(\frac{q}{x_{i}} ; q\right)_{b} \prod_{1 \leq i<j \leq n}\left(\frac{x_{i}}{x_{j}}, \frac{q x_{j}}{x_{i}} ; q\right)_{k}\right]
\end{aligned}
$$

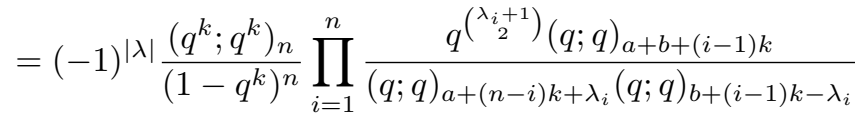

$$
\begin{aligned}
& \times \prod_{1 \leq i<j \leq n}\left(q^{(j-i) k+\lambda_{i}-\lambda_{j}} ; q\right)_{k} .
\end{aligned}
$$


Here $a, b \geq 0,|\lambda|=\sum_{i=1}^{n} \lambda_{i},\left(a_{1}, \ldots, a_{r} ; q\right)_{m}=\prod_{j=1}^{r}\left(a_{j} ; q\right)_{m}, 1 /(q ; q)_{-m}=0$ for $m$ a positive integer, and C.T. $[f(x)]$ denotes the constant term of the Laurent polynomial $f(x)$. Kaneko's constant term identity has a rich history. For $\lambda=0$ it was conjectured by Morris [23, Conjecture (4.12)] who proved his conjecture for $q=1$ [23, Theorem (4.13)]. $q$-Proofs for $\lambda=0$ were first obtained by Kadell [12, Theorem $3 ; l=m=0$ ] and Habsieger [9, p. 1487] based upon their Askey-KadellHabsieger integral evaluation. For general $\lambda$ but $q=1$ the above identity was first established by Kadell [14, Equation (A)] (see also [13, Theorem 4]).

Taking Theorem 1.4 as starting point we will prove the following generalization of the $b=k-a-1$ case of Kaneko's constant term identity.

Theorem 1.7. Let $\lambda=\left(\lambda_{1}, \ldots, \lambda_{n}\right)$ and $\mu=\left(\mu_{1}, \ldots, \mu_{n}\right)$ be partitions, and $a$ and $k$ integers such that $a \in\{0, \ldots, k-1\}$. Then

$$
\begin{array}{r}
\text { C.T. }\left[P_{\lambda}\left(x ; q, q^{k}\right) P_{\mu}\left(x^{-1} ; q, q^{k}\right) \prod_{i=1}^{n}\left(x_{i} ; q\right)_{a}\left(\frac{q}{x_{i}} ; q\right)_{k-a-1} \prod_{1 \leq i<j \leq n}\left(\frac{x_{i}}{x_{j}}, \frac{q x_{j}}{x_{i}} ; q\right)_{k}\right] \\
\left.=(-1)^{|\lambda|+|\mu|} \frac{\left(q^{k} ; q^{k}\right)_{n}}{\left(1-q^{k}\right)^{n}} \prod_{i=1}^{n} q^{\left(\mu_{i}-\lambda_{i}\right.}\right)\left[\begin{array}{c}
k-1 \\
a+\lambda_{i}-\mu_{i}
\end{array}\right]_{q} \\
\times \prod_{1 \leq i<j \leq n} \frac{\left(q^{(j-i) k+\lambda_{i}-\lambda_{j}} ; q\right)_{k}\left(q^{(j-i) k+\mu_{i}-\mu_{j}} ; q\right)_{k}}{\left(q^{1+a-k+(j-i) k+\lambda_{i}-\mu_{j}} ; q\right)_{k}\left(q^{-a+(j-i) k+\mu_{i}-\lambda_{j}} ; q\right)_{k}} .
\end{array}
$$

Here $f\left(x^{-1}\right)=f\left(x_{1}^{-1}, \ldots, x_{n}^{-1}\right)$ and

$$
\left[\begin{array}{l}
r \\
a
\end{array}\right]_{q}= \begin{cases}\frac{(q ; q)_{r}}{(q ; q)_{a}(q ; q)_{r-a}} & a \in\{0, \ldots, r\} \\
0 & \text { otherwise }\end{cases}
$$

is a $q$-binomial coefficient.

The correct interpretation of the statement of the theorem is that the constant term is zero whenever the $q$-binomial coefficient on the right vanishes, i.e., whenever $a+\lambda_{i}-\mu_{i} \notin\{0, \ldots, k-1\}$ for some $i \in\{1, \ldots, n\}$. This convention makes the right-hand side well-defined for all partitions $\lambda$ and $\mu$ as we shall now show.

The double product on the right has a zero factor in the denominator if

$$
a+(j-i) k+\lambda_{i}-\mu_{j} \in\{0, \ldots, k-1\} \text { for } i, j \in\{1, \ldots, n\}, i \neq j .
$$

Now assume that for given $i$ and $j$ (1.11) holds. By adding and subtracting $\lambda_{j}$, this implies $(j-i) k+\left(\lambda_{i}-\lambda_{j}\right)+\left(a+\lambda_{j}-\mu_{j}\right) \in\{0, \ldots, k-1\}$. For $i<j$ we have $(j-i) k+\lambda_{i}-\lambda_{j} \geq k$ so that $a+\lambda_{j}-\mu_{j}$ must be negative. If $i>j$ we have $(j-i) k+\lambda_{i}-\lambda_{j} \leq-k$ so that $a+\lambda_{j}-\mu_{j}$ must be greater or equal to $k$. We thus conclude that zero factors in the denominator cannot occur when the $q$-binomial coefficient on the right is non-vanishing. Hence the constant term is either zero or a well-defined rational function.

We further note that the theorem is invariant under the simultaneous replacements $a \mapsto k-1-a$ and $\lambda \leftrightarrow \mu$. On the left side this requires the change $t_{i} \mapsto q / t_{n-i+1}$, and the symmetry and homogeneity of the Macdonald polynomials, see Section 2. In the proof of the theorem we will in fact show the stronger statement that the non-zero values of $a$ follow from $a=0$.

By letting $q$ tend to 1 in Theorem 1.7 we obtain a constant term identity of Kadell [13, Theorem 5]. 
Corollary 1.8. With the same conditions as in Theorem 1.7 there holds

$$
\begin{aligned}
& \text { C.T. } {\left[P_{\lambda}^{(1 / k)}(t) P_{\mu}^{(1 / k)}\left(t^{-1}\right) \prod_{i=1}^{n}\left(1-t_{i}\right)^{a}\left(1-\frac{1}{t_{i}}\right)^{k-a-1} \prod_{1 \leq i<j \leq n}\left(1-\frac{t_{i}}{t_{j}}\right)^{k}\left(1-\frac{t_{j}}{t_{i}}\right)^{k}\right] } \\
&=n !(-1)^{|\lambda|+|\mu|} \prod_{i=1}^{n}\left(\begin{array}{c}
k-1 \\
a+\lambda_{i}-\mu_{i}
\end{array}\right) \\
& \quad \times \prod_{1 \leq i<j \leq n} \frac{\left((j-i) k+\lambda_{i}-\lambda_{j}\right)_{k}\left((j-i) k+\mu_{i}-\mu_{j}\right)_{k}}{\left(1+a-k+(j-i) k+\lambda_{i}-\mu_{j}\right)_{k}\left(-a+(j-i) k+\mu_{i}-\lambda_{j}\right)_{k}} .
\end{aligned}
$$

In Section 9 of Chapter VI of his book [20], Macdonald defines a scalar product on $L_{n}$, the $\mathbb{Q}(q, t)$-algebra of Laurent polynomials in $x$. When $t=q^{k}$ this scalar product reads

$$
\langle f, g\rangle^{\prime}=\frac{1}{n !} \text { C.T. }\left[f(x) g\left(x^{-1}\right) \prod_{1 \leq i<j \leq n}\left(\frac{x_{i}}{x_{j}}, \frac{x_{j}}{x_{i}} ; q\right)_{k}\right],
$$

for $f, g \in L_{n}$. A straightforward symmetrization (see Lemma 3.2) shows that Theorem 1.7 is equivalent to

$$
\begin{aligned}
& \left\langle P_{\lambda} h_{a, k}, P_{\mu}\right\rangle^{\prime}=(-1)^{|\lambda|+|\mu|} \prod_{i=1}^{n} q^{\left(\mu_{i}-\lambda_{i}\right)}\left[\begin{array}{c}
k-1 \\
a+\lambda_{i}-\mu_{i}
\end{array}\right]_{q} \\
& \times \prod_{1 \leq i<j \leq n} \frac{\left(q^{(j-i) k+\lambda_{i}-\lambda_{j}} ; q\right)_{k}\left(q^{(j-i) k+\mu_{i}-\mu_{j}} ; q\right)_{k}}{\left(q^{1+a-k+(j-i) k+\lambda_{i}-\mu_{j}} ; q\right)_{k}\left(q^{-a+(j-i) k+\mu_{i}-\lambda_{j}} ; q\right)_{k}},
\end{aligned}
$$

for

$$
h_{a, k}(x)=\prod_{i=1}^{n}\left(x_{i} ; q\right)_{a}\left(\frac{q}{x_{i}} ; q\right)_{k-a-1} .
$$

Following Kadell's treatment of the case $q=1$ [13], we will show that this can be viewed as a generalization of Macdonald's orthogonality relation and norm evaluation for Macdonald polynomials with respect to the scalar product (1.12), which is given by 20, Example VI.9.1(d)]

$$
\left\langle P_{\lambda}, P_{\mu}\right\rangle^{\prime}=\delta_{\lambda \mu} \prod_{1 \leq i<j \leq n} \frac{\left(q^{(j-i) k+\lambda_{i}-\lambda_{j}} ; q\right)_{k}}{\left(q^{1-k+(j-i) k+\lambda_{i}-\lambda_{j}} ; q\right)_{k}} .
$$

Still following Kadell [13. Theorem 8], we will then show that Theorem 1.7 implies the following $q$-binomial theorem for Macdonald polynomials.

Corollary 1.9. Let $\mu=\left(\mu_{1}, \ldots, \mu_{n}\right)$ be a partition and $k$ a positive integer. Then

$$
\sum_{\lambda} c_{\lambda \mu}^{k}(q) P_{\lambda}\left(x ; q, q^{k}\right)=P_{\mu}\left(x ; q, q^{k}\right) \prod_{i=1}^{n}\left(x_{i} ; q\right)_{k-1},
$$

where

$$
\begin{aligned}
c_{\lambda \mu}^{k}(q)=(-1)^{|\lambda|+|\mu|} & \left.\prod_{i=1}^{n} q^{\left(\lambda_{i}-\mu_{i}\right.}\right)\left[\begin{array}{c}
k-1 \\
\lambda_{i}-\mu_{i}
\end{array}\right]_{q} \\
& \times \prod_{1 \leq i<j \leq n} \frac{\left(q^{(j-i) k+\mu_{i}-\mu_{j}} ; q\right)_{k}\left(q^{1-k+(j-i) k+\lambda_{i}-\lambda_{j}} ; q\right)_{k}}{\left(q^{(j-i) k+\mu_{i}-\lambda_{j}} ; q\right)_{k}\left(q^{1-k+(j-i) k+\lambda_{i}-\mu_{j}} ; q\right)_{k}} .
\end{aligned}
$$


Here we follow the same convention as in Theorem 1.7 in that $c_{\lambda \mu}^{k}(q)=0$ unless $\lambda_{i}-\mu_{i} \in\{0, \ldots, k-1\}$ for all $i \in\{1, \ldots, n\}$. A slightly more general result is given in Corollary 3.3

Finally, using an identity for the structure constants of the Macdonald polynomials, we generalize Corollary 1.9 from $t=q^{k}$ to all $t$.

Theorem 1.10. If $\mu=\left(\mu_{1}, \ldots, \mu_{n}\right)$ is a partition then

$$
\sum_{\lambda} \frac{\left(q t^{n-1}\right)_{\lambda} P_{\lambda}(x)}{c_{\lambda}^{\prime}} \prod_{i, j=1}^{n} \frac{\left(q t^{j-i-1} ; q\right)_{\lambda_{i}-\mu_{j}}}{\left(q t^{j-i} ; q\right)_{\lambda_{i}-\mu_{j}}}=t^{(n-1)|\mu|} \frac{P_{\mu}(x)}{P_{\mu}\left(t^{\delta}\right)} \prod_{i=1}^{n} \frac{\left(x_{i} q / t ; q\right)_{\infty}}{\left(x_{i} ; q\right)_{\infty}}
$$

for $|q|<1$ and $\max \left\{\left|x_{i}\right|\right\}_{i=1}^{n}<1$.

Here

$$
(a)_{\lambda}=(a ; q, t)_{\lambda}=t^{n(\lambda)} \prod_{i=1}^{n}\left(a t^{1-i} ; q\right)_{\lambda_{i}}
$$

is a $q$-shifted factorial labelled by the partition $\lambda$ and

$$
c_{\lambda}^{\prime}=c_{\lambda}^{\prime}(q, t)=t^{-n(\lambda)}\left(q t^{n-1}\right)_{\lambda} \prod_{1 \leq i<j \leq n} \frac{\left(q t^{j-i-1} ; q\right)_{\lambda_{i}-\lambda_{j}}}{\left(q t^{j-i} ; q\right)_{\lambda_{i}-\lambda_{j}}}
$$

is a generalized hook polynomial. Since the summand on the left vanishes when $\lambda \not \supset \mu$, i.e., when the partition $\mu$ is not contained in the partition $\lambda\left(\lambda_{i} \geq \mu_{i}\right.$ for all $i$ ), the sum over $\lambda$ may be replaced by a sum over $\lambda$ subject to $\lambda \supset \mu$. When $\mu=0$ the double product in the summand on the left becomes $(q / t)_{\lambda} /\left(q t^{n-1}\right)_{\lambda}$ and Theorem 1.10 reduces to a special case of the $q$-binomial theorem for Macdonald polynomials given by 2.17).

In the next section we present some necessary background material on Macdonald polynomials and in Sections 3.1 3.6 we prove all of the claims made in the introduction. Finally, in Section 4 we conclude with an (ex)-conjecture of Askey closely related to the Askey-Habsieger-Kadell integral.

\section{Preliminaries on Macdonald polynomials}

We review some of the theory of Macdonald polynomials needed for our subsequent proofs. For more details we refer to [20, Chapter VI].

Let $\lambda=\left(\lambda_{1}, \lambda_{2}, \ldots\right)$ be a partition, i.e., $\lambda_{1} \geq \lambda_{2} \geq \ldots$ with finitely many $\lambda_{i}$ unequal to zero. The length and weight of $\lambda$, denoted by $\ell(\lambda)$ and $|\lambda|$, are the number and sum of the non-zero $\lambda_{i}$, respectively. We shall not employ the frequency or multiplicity notation for partitions except for partitions of rectangular shape, in which case we abbreviate $(N, \ldots, N)$ with $n$ repeated $N$ s as $\left(N^{n}\right)$. For $\lambda$ a partition and $a$ an integer we write

$$
\lambda \pm a=\left(\lambda_{1} \pm a, \lambda_{2} \pm a, \ldots\right) .
$$

For two partitions $\lambda$ and $\mu$ we define the usual addition as $\lambda+\mu=\left(\lambda_{1}+\mu_{1}, \lambda_{2}+\right.$ $\left.\mu_{2}, \ldots\right)$, and for $x=\left(x_{1}, x_{2}, \ldots\right)$ a sequence (possibly a partition) and $z$ a scalar, we set $z x=\left(z x_{1}, z x_{2}, \ldots\right)$.

The dominance partial order on the set of partitions is defined as follows: $\lambda \geq \mu$ if and only if $|\lambda|=|\mu|$ and $\lambda_{1}+\cdots+\lambda_{i} \geq \mu_{1}+\cdots+\mu_{i}$ for all $i \geq 1$.

As usual we identify a partition with its diagram or Ferrers graph, defined by the set of points in $(i, j) \in \mathbb{Z}^{2}$ such that $1 \leq j \leq \lambda_{i}$. The conjugate $\lambda^{\prime}$ of $\lambda$ is the 
partition obtained by reflecting the diagram of $\lambda$ in the main diagonal. If $\lambda$ and $\mu$ are partitions then $\lambda \supset \mu$ means that (the diagram of) $\mu$ is contained in (the diagram of) $\lambda$, i.e., $\lambda_{i} \geq \mu_{i}$ for all $i \geq 1$. Let $s=(i, j)$ be a square in the diagram of $\lambda$. Then $a(s), a^{\prime}(s), l(s)$ and $l^{\prime}(s)$ are the arm-length, arm-colength, leg-length and leg-colength of $s$, defined by

$$
\begin{aligned}
a(s) & =\lambda_{i}-j, & a^{\prime}(s) & =j-1 \\
l(s) & =\lambda_{j}^{\prime}-i, & l^{\prime}(s) & =i-1 .
\end{aligned}
$$

An often used statistic on partitions is

$$
n(\lambda)=\sum_{i \geq 1}(i-1) \lambda_{i}=\sum_{i \geq 1}\left(\begin{array}{c}
\lambda_{i}^{\prime} \\
2
\end{array}\right) .
$$

In the following we will almost exclusively work with partitions $\lambda=\left(\lambda_{1}, \ldots, \lambda_{n}\right)$ with $\ell(\lambda) \leq n$. For such a partition the monomial symmetric function $m_{\lambda}(x)$ in the variables $x=\left(x_{1}, \ldots, x_{n}\right)$ is defined as

$$
m_{\lambda}(x)=\sum x^{\alpha}
$$

where the sum is over all distinct permutations $\alpha$ of $\lambda$, and $x^{\alpha}=x_{1}^{\alpha_{1}} \cdots x_{n}^{\alpha_{n}}$. The monomial symmetric functions form a $\mathbb{Z}$-basis of $\Lambda_{n}$, the ring of symmetric polynomials in $x_{1}, \ldots, x_{n}$ with integer coefficients.

The $r$-th power sum $p_{r}$ is given by $p_{r}(x)=m_{(r)}(x)=\sum_{i=1}^{n} x_{i}^{n}$, and, more generally, the power-sum products are defined as $p_{\lambda}(x)=p_{\lambda_{1}}(x) \ldots p_{\lambda_{n}}(x)$. Following Macdonald we now introduce the scalar product $\langle\cdot, \cdot\rangle$ on $\Lambda_{n}$ by

$$
\left\langle p_{\lambda}, p_{\mu}\right\rangle=\delta_{\lambda \mu} z_{\lambda} \prod_{i=1}^{n} \frac{1-q^{\lambda_{i}}}{1-t^{\lambda_{i}}},
$$

with $z_{\lambda}=\prod_{i \geq 1} m_{i} ! i^{m_{i}}, m_{i}$ being the number of parts of $\lambda$ equal to $i$.

Denote the ring of symmetric functions in $n$ variables over the field $F=\mathbb{Q}(q, t)$ of rational functions in $q$ and $t$ by $\Lambda_{n, F}$. Then the Macdonald polynomial $P_{\lambda}(x ; q, t)$ is the unique symmetric polynomial in $\Lambda_{n, F}$ such that [20, Equation (VI.4.7)]:

$$
P_{\lambda}=\sum_{\mu \leq \lambda} u_{\lambda \mu} m_{\mu}
$$

where $u_{\lambda \mu} \in F, u_{\lambda \lambda}=1$, and

$$
\left\langle P_{\lambda}, P_{\mu}\right\rangle=0 \text { if } \lambda \neq \mu \text {. }
$$

The Macdonald polynomials form an $F$-basis of $\Lambda_{n, F}$. For $t=1$ and $t=q$ they reduce to the monomial symmetric functions and Schur functions; $P_{\lambda}(x ; q, 1)=$ $m_{\lambda}(x)$ and $P_{\lambda}(x ; q, q)=s_{\lambda}(x)$. More generally,

$$
\lim _{t \rightarrow 1} P_{\lambda}\left(x ; t^{\alpha}, t\right)=P_{\lambda}^{(\alpha)}(x)
$$

with $P_{\lambda}^{(\alpha)}$ Jack's symmetric function.

Below we have listed several known results for Macdonald polynomials that will be needed subsequently. $P_{\lambda}$ is homogeneous of degree $|\lambda|$, i.e., for $z$ a scalar,

$$
P_{\lambda}(z x)=z^{|\lambda|} P_{\lambda}(x) .
$$

This is of course immediate from 2.3) and 2.4. Also, [20, Equation (VI.4.17)]

$$
P_{\lambda+a}(x)=\left(x_{1} \ldots x_{n}\right)^{a} P_{\lambda}(x),
$$


provided (for the time being) that $\lambda_{n}+a$ is a nonnegative integer so that both sides are well-defined.

Recalling 2.2), we introduce two generalized hook polynomials as [20, Equation (VI.8.1)]

$$
\begin{aligned}
& c_{\lambda}=c_{\lambda}(q, t)=\prod_{s \in \lambda}\left(1-q^{a(s)} t^{l(s)+1}\right) \\
& c_{\lambda}^{\prime}=c_{\lambda}^{\prime}(q, t)=\prod_{s \in \lambda}\left(1-q^{a(s)+1} t^{l(s)}\right)
\end{aligned}
$$

and set

$$
b_{\lambda}=b_{\lambda}(q, t)=\frac{c_{\lambda}(q, t)}{c_{\lambda}^{\prime}(q, t)} .
$$

The quadratic norm evaluation of the Macdonald polynomials can be very simply expressed in terms of this last function as [20, Equation (VI.6.19)]

$$
\left\langle P_{\lambda}, P_{\lambda}\right\rangle^{-1}=b_{\lambda}
$$

Using a generalized $q$-shifted factorial

$$
(a)_{\lambda}=(a ; q, t)_{\lambda}=\prod_{s \in \lambda}\left(t^{l^{\prime}(s)}-a q^{a^{\prime}(s)}\right)=t^{n(\lambda)} \prod_{i=1}^{n}\left(a t^{1-i} ; q\right)_{\lambda_{i}}
$$

we explicitly have [16, Proposition 3.2]

$$
\begin{aligned}
& c_{\lambda}=t^{-n(\lambda)}\left(t^{n}\right)_{\lambda} \prod_{1 \leq i<j \leq n} \frac{\left(t^{j-i} ; q\right)_{\lambda_{i}-\lambda_{j}}}{\left(t^{j-i+1} ; q\right)_{\lambda_{i}-\lambda_{j}}} \\
& c_{\lambda}^{\prime}=t^{-n(\lambda)}\left(q t^{n-1}\right)_{\lambda} \prod_{1 \leq i<j \leq n} \frac{\left(q t^{j-i-1} ; q\right)_{\lambda_{i}-\lambda_{j}}}{\left(q t^{j-i} ; q\right)_{\lambda_{i}-\lambda_{j}}} .
\end{aligned}
$$

The $b_{\lambda}$ also features in the Cauchy identity for Macdonald polynomials. Defining $y=\left(y_{1}, \ldots, y_{n}\right)$ in addition to the usual $x=\left(x_{1}, \ldots, x_{n}\right)$, this identity states that [20, Equation (VI.4.13)]

$$
\sum_{\lambda} b_{\lambda} P_{\lambda}(x) P_{\lambda}(y)=\prod_{i, j=1}^{n} \frac{\left(t x_{i} y_{j} ; q\right)_{\infty}}{\left(x_{i} y_{j} ; q\right)_{\infty}} .
$$

This may be alternatively put in terms of $y=\left(y_{1}, \ldots, y_{m}\right)$ as [20, Equation (VI.5.4)]

$$
\sum_{\substack{\lambda \\ \lambda_{1} \leq m}} P_{\lambda}(x ; q, t) P_{\lambda^{\prime}}(y ; t, q)=\prod_{\substack{1 \leq i \leq n \\ 1 \leq j \leq m}}\left(1+x_{i} y_{j}\right) .
$$

Next we need the evaluation homomorphism $u_{\lambda}: \Lambda_{n, F} \rightarrow F$ defined by

$$
u_{\lambda}(f)=f\left(q^{\lambda} t^{\delta}\right)
$$

for $f \in \Lambda_{n, F}$. Here $q^{\lambda} t^{\delta}=\left(q^{\lambda_{1}} t^{\delta_{1}}, q^{\lambda_{2}} t^{\delta_{2}}, \ldots, q^{\lambda_{n}} t^{\delta_{n}}\right)$, with $\delta$ denoting the special partition $\delta=(n-1, n-2, \ldots, 1,0)$. Two very important results that will be frequently used are the specialization [20, Example VI.6.5]

$$
u_{0}\left(P_{\lambda}\right)=P_{\lambda}\left(t^{\delta}\right)=P_{\lambda}\left(1, t, \ldots, t^{n-1}\right)=t^{n(\lambda)} \prod_{s \in \lambda} \frac{1-q^{a^{\prime}(s)} t^{n-l^{\prime}(s)}}{1-q^{a(s)} t^{l(s)+1}}
$$


or, by 2.7a), 2.9 and 2.10a, 20, Example VI.6.5]

$$
\begin{aligned}
P_{\lambda}\left(t^{\delta}\right) & =\frac{\left(t^{n}\right)_{\lambda}}{c_{\lambda}} \\
& =t^{n(\lambda)} \prod_{1 \leq i<j \leq n} \frac{\left(t^{j-i+1} ; q\right)_{\lambda_{i}-\lambda_{j}}}{\left(t^{j-i} ; q\right)_{\lambda_{i}-\lambda_{j}}},
\end{aligned}
$$

and the symmetry [20, Equation (VI.6.6)]

$$
u_{\lambda}\left(P_{\mu}\right) u_{0}\left(P_{\lambda}\right)=u_{\mu}\left(P_{\lambda}\right) u_{0}\left(P_{\mu}\right) .
$$

Finally we follow Kaneko [16] and Macdonald 21] in defining multivariable basic hypergeometric series and multivariable bilateral basic hypergeometric series with Macdonald polynomial argument. Extending the condensed notation for $q$-shifted factorials to the multivariable setting by

$$
\left(a_{1}, \ldots, a_{r}\right)_{\lambda}=\left(a_{1}, \ldots, a_{r} ; q, t\right)_{\lambda}=\prod_{j=1}^{r}\left(a_{j} ; q, t\right)_{\lambda}=\prod_{j=1}^{r}\left(a_{j}\right)_{\lambda}
$$

we define

$$
{ }_{r+1} \Phi_{r}\left[\begin{array}{c}
a_{1}, \ldots, a_{r+1} \\
b_{1}, \ldots, b_{r}
\end{array} ; q, t, x\right]=\sum_{\lambda} \frac{\left(a_{1}, \ldots, a_{r+1}\right)_{\lambda}}{\left(b_{1}, \ldots, b_{r}\right)_{\lambda} c_{\lambda}^{\prime}} P_{\lambda}(x) .
$$

Often we employ the one-line notation ${ }_{r+1} \Phi_{r}\left(a_{1}, \ldots, a_{r+1} ; b_{1}, \ldots, b_{r} ; q, t, x\right)$ for the above series. For $n=1$ we have $P_{(k)}(x)=x^{k}$ and $c_{(k)}^{\prime}=(q ; q)_{k}$ so that the ${ }_{r+1} \Phi_{r}$ series simplifies to the standard one-variable basic hypergeometric series $\left.{ }_{r+1} \phi_{r} 8\right]$.

An important result needed a number of times is the following multivariable version of the $q$-binomial theorem due to Kaneko [16, Theorem 3.5] and Macdonald [21, Equation (2.2)]:

$$
{ }_{1} \Phi_{0}(a ;-; q, t, x)=\prod_{i=1}^{n} \frac{\left(a x_{i} ; q\right)_{\infty}}{\left(x_{i} ; q\right)_{\infty}},
$$

for $|q|<1$ and $\max \left\{\left|x_{i}\right|\right\}_{i=1}^{n}<1$. In the Jack polynomial case this was also found by Yan [26, Proposition 3.1] and in the Schur polynomial case (Jack with $\alpha=1$ ) by Milne [22, Theorem 1.18]. When $a=q^{-N}$ with $N$ a nonnegative integer the series on the left of 2.17) terminates leading to

$$
{ }_{1} \Phi_{0}\left(q^{-N} ;-; q, t, x\right)=\prod_{i=1}^{n}\left(x_{i} q^{-N} ; q\right){ }_{N} .
$$

We also need the generalization of ${ }_{r+1} \Phi_{r}$ to bilateral series. Let us denote the set of weakly decreasing integer sequences of length $n$ by $P$. The relation 1.8 (which follows from (2.6) by the substitutions $\left.\lambda \mapsto \lambda-\left(\lambda_{n}, \ldots, \lambda_{n}\right), a \mapsto \lambda_{n}\right)$ can be used to define the Macdonald polynomial for all $\lambda \in P$. By extending the definition of the $q$-shifted factorials to all integers $n$ by

$$
(a ; q)_{n}=\frac{(a ; q)_{\infty}}{\left(a q^{n} ; q\right)_{\infty}}
$$

we can also give meaning to $(a)_{\lambda}$ of 2.9$)$ and $c_{\lambda}^{\prime}$ of $2.10 \mathrm{~b}$ for $\lambda \in P$. Observe that $1 /(q ; q)_{-n}=0$ for $n$ a positive integer and hence that $1 /\left(q t^{n-1}\right)_{\lambda}=0$ if $\lambda$ is not a partition. However, $\left(q t^{n-1}\right)_{\lambda} / c_{\lambda}^{\prime}$ is perfectly well-defined for all $\lambda \in P$. We 
now use the above definitions to define the bilateral multiple basic hypergeometric series

$$
{ }_{r} \Psi_{r}\left[\begin{array}{l}
a_{1}, \ldots, a_{r} \\
b_{1}, \ldots, b_{r}
\end{array} ; q, t, x\right]=\sum_{\lambda \in P} \frac{\left(q t^{n-1}, a_{1}, \ldots, a_{r}\right)_{\lambda}}{\left(b_{1}, \ldots, b_{r}\right)_{\lambda} c_{\lambda}^{\prime}} P_{\lambda}(x) .
$$

Again we also employ the one-line notation ${ }_{r} \Psi_{r}\left(a_{1}, \ldots, a_{r} ; b_{1}, \ldots, b_{r} ; q, t, x\right)$. We remark that we have chosen a slightly different (more symmetrical) form for ${ }_{r} \Psi_{r}$ compared to earlier definitions of Milne for $t=q[22$ ] and of Kaneko [18] for general $t$. In view of our earlier comments, the series 2.20$)$ simplifies to the unilateral series (2.16) with $r \mapsto r-1$ if $b_{r}=q t^{n-1}$. For $n=1$ the series 2.20 reduces to the classical bilateral basic hypergeometric series ${ }_{r} \psi_{r} 8$.

Kaneko [18, Equation (1.11)] proved the following multiple series analogue of Ramanujan's celebrated ${ }_{1} \psi_{1}$ summation formula:

$$
{ }_{1} \Psi_{1}(a ; b ; q, t, x)=\prod_{i=1}^{n} \frac{\left(q t^{n-i}, b t^{1-i} / a, a x_{i}, q / a x_{i} ; q\right)_{\infty}}{\left(b t^{1-i}, q t^{n-i} / a, x_{i}, b t^{1-n} / a x_{i} ; q\right)_{\infty}}
$$

for $\left|b t^{1-n} / a\right|<\left|x_{i}\right|<1$ for all $i \in\{1, \ldots, n\}$. Kaneko's proof utilizes the $q$-binomial theorem 2.17) (obtained for $b=q t^{n-1}$ ) and Ismail's analytic argument. A different proof based on a Gauss summation for Macdonald polynomials was subsequently found by Baker and Forrester [6, Theorem 6.1]. We should also mention that the Schur case of the theorem, corresponding to $t=q$, was first obtained by Milne using a determinant evaluation [22, Theorem 1.14].

A last comment concerning the Macdonald polynomials $P_{\lambda}$ for $\lambda \in P$ is that the relations (2.5), 2.6) and (2.15) still hold. In the case of (2.6) we may now in fact assume that $\lambda_{n}+a$ is any integer. In the case of (2.5),

$$
\begin{aligned}
P_{\lambda}(z x) & =\left(z^{n} x_{1} \ldots, x_{n}\right)^{\lambda_{n}} P_{\lambda-\lambda_{n}}(z x) \\
& =z^{|\lambda|}\left(x_{1} \ldots, x_{n}\right)^{\lambda_{n}} P_{\lambda-\lambda_{n}}(x)=z^{|\lambda|} P_{\lambda}(x)
\end{aligned}
$$

by (1.8), then (2.5), and then (1.8). For $(2.6)$ the above claim is evident as both sides may be rewritten by 1.8 as $\left(x_{1} \ldots x_{n}\right)^{\lambda_{n}+a} P_{\lambda-\lambda_{n}}(x)$. Finally, in the case of 2.15 ,

$$
\begin{aligned}
u_{\lambda}\left(P_{\mu}\right) u_{0}\left(P_{\lambda}\right) & =q^{\mu_{n}|\lambda|} t^{\left(\lambda_{n}+\mu_{n}\right)\left(\begin{array}{c}
n \\
2
\end{array}\right)} u_{\lambda}\left(P_{\mu-\mu_{n}}\right) u_{0}\left(P_{\lambda-\lambda_{n}}\right) \\
& =q^{\mu_{n}|\lambda|+\lambda_{n}|\mu|-n \lambda_{n} \mu_{n}} t^{\left(\lambda_{n}+\mu_{n}\right)\left(\begin{array}{l}
n \\
2
\end{array}\right)} u_{\lambda-\lambda_{n}}\left(P_{\mu-\mu_{n}}\right) u_{0}\left(P_{\lambda-\lambda_{n}}\right) \\
& =q^{\mu_{n}|\lambda|+\lambda_{n}|\mu|-n \lambda_{n} \mu_{n}} t^{\left(\lambda_{n}+\mu_{n}\right)\left(\begin{array}{l}
n \\
2
\end{array}\right)} u_{\mu-\mu_{n}}\left(P_{\lambda-\lambda_{n}}\right) u_{0}\left(P_{\mu-\mu_{n}}\right) \\
& =\cdots=u_{\mu}\left(P_{\lambda}\right) u_{0}\left(P_{\mu}\right) .
\end{aligned}
$$

by (1.8), then (2.5), then (2.15), et cetera.

\section{Proofs}

3.1. Proof of Theorem 1.1. We take the ${ }_{1} \Psi_{1}$ sum 2.21 , replace $x \mapsto z x$ with $z$ a scalar and use 2.5). Then we apply the evaluation homomorphism $u_{\mu}$ defined in (2.13) and utilize the symmetry 2.15) to eliminate $u_{\mu}\left(P_{\lambda}\right)$, with $\lambda$ the summation 
index of the ${ }_{1} \Psi_{1}$ series. Hence

$$
\begin{aligned}
& \sum_{\lambda \in P} \frac{\left(q t^{n-1}, a\right)_{\lambda} z^{|\lambda|}}{(b)_{\lambda} c_{\lambda}^{\prime}} \frac{u_{\lambda}\left(P_{\mu}\right) u_{0}\left(P_{\lambda}\right)}{u_{0}\left(P_{\mu}\right)} \\
&=\prod_{i=1}^{n} \frac{\left(q t^{n-i}, b t^{1-i} / a, a z q^{\mu_{i}} t^{n-i}, q^{1-\mu_{i}} t^{i-n} / a z ; q\right)_{\infty}}{\left(b t^{1-i}, q t^{n-i} / a, z q^{\mu_{i}} t^{n-i}, b q^{-\mu_{i}} t^{i-2 n+1} / a z ; q\right)_{\infty}} .
\end{aligned}
$$

To transform this into Theorem 1.1 we need to specialize

$$
t=q^{k}, \quad z=q^{\alpha}, \quad a=-c q^{(n-1) k}, \quad b=-c q^{\alpha+\beta+3(n-1) k} .
$$

Using (1.7) and 2.10b in the form

$$
c_{\lambda}^{\prime}\left(q, q^{k}\right)=q^{-k n(\lambda)}\left(q^{1+(n-1) k} ; q, q^{k}\right)_{\lambda} \prod_{1 \leq i<j \leq n} \frac{\left(q^{1+(j-i-1) k} ; q\right)_{k}}{\left(q^{1+(j-i-1) k+\lambda_{i}-\lambda_{j}} ; q\right)_{k}},
$$

and noting that

$$
(a ; q)_{k}\left(a q^{k} ; q\right)_{n}=(a ; q)_{k+n}
$$

yields

$$
\begin{aligned}
\frac{u_{0}\left(P_{\lambda}\right)}{c_{\lambda}^{\prime}\left(q, q^{k}\right)}= & \frac{q^{2 k n(\lambda)}}{\left(q^{1+(n-1) k} ; q, q^{k}\right)_{\lambda}} \\
& \times \prod_{1 \leq i<j \leq n} \frac{1-q^{(j-i) k+\lambda_{i}-\lambda_{j}}}{1-q^{(j-i) k}} \frac{\left(q^{1+(j-i-1) k+\lambda_{i}-\lambda_{j}} ; q\right)_{2 k-1}}{\left(q^{1+(j-i-1) k} ; q\right)_{2 k-1}} \\
= & \frac{q^{2 k n(\lambda)}}{\left(q^{1+(n-1) k} ; q, q^{k}\right)_{\lambda}} \prod_{i=1}^{n} \frac{1-q^{i k}}{1-q^{k}} \frac{(q ; q)_{k}}{(q ; q)_{(i-1) k}(q ; q)_{i k}} \\
& \times \prod_{1 \leq i<j \leq n}\left(1-q^{(j-i) k+\lambda_{i}-\lambda_{j}}\right)\left(q^{1+(j-i-1) k+\lambda_{i}-\lambda_{j}} ; q\right)_{2 k-1},
\end{aligned}
$$

where here and in the following $P_{\lambda}(x)=P_{\lambda}\left(x ; q, q^{k}\right)$. Employing the definitions (1.2) and (1.3) of the $q$-gamma function and theta function, we thus obtain the identity

$$
\begin{gathered}
\sum_{\lambda \in P} q^{\alpha|\lambda|+2 k n(\lambda)} \frac{u_{\lambda}\left(P_{\mu}\right)}{u_{0}\left(P_{\mu}\right)} \prod_{i=1}^{n} \frac{\left(-c q^{\alpha+\beta+(3 n-i-2) k+\lambda_{i}} ; q\right)_{\infty}}{\left(-c q^{(n-i) k+\lambda_{i}} ; q\right)_{\infty}} \\
\times \prod_{1 \leq i<j \leq n}\left(1-q^{(j-i) k+\lambda_{i}-\lambda_{j}}\right)\left(q^{1+(j-i-1) k+\lambda_{i}-\lambda_{j}} ; q\right)_{2 k-1} \\
=\prod_{i=1}^{n} \frac{1-q^{k}}{1-q^{i k}} \frac{\Gamma_{q}\left(\alpha+(n-i) k+\mu_{i}\right) \Gamma_{q}\left(\beta+(i-1) k-\mu_{i}\right) \Gamma_{q}(i k+1)}{\Gamma_{q}(\alpha+\beta+(n+i-2) k) \Gamma_{q}(k+1)} \\
\times \frac{\vartheta\left(-c q^{\alpha+(2 n-i-1) k+\mu_{i}} ; q\right)}{\vartheta\left(-c q^{(n-i) k} ; q\right)} .
\end{gathered}
$$

The next step of the proof relies on the following lemma. Let $S_{n}$ be the symmetric group, with elements $w$ given by the permutations of $(1, \ldots, n)$. $S_{n}$ acts on $f(x)$ as $w(f(x))=f(w(x))=f\left(x_{w_{1}}, \ldots, x_{w_{n}}\right)$.

Lemma 3.1. For $k$ a positive integer and $w \in S_{n}$ let

$$
h_{\lambda}=h_{w(\lambda)}
$$


and

$$
h_{\lambda}=0 \quad \text { if } \quad\left|\lambda_{i}-\lambda_{j}\right| \in\{0, \ldots, k-1\} .
$$

for all $1 \leq i<j \leq n$. Then, formally,

$$
\sum_{\lambda \in P} h_{\lambda+k \delta}=\frac{(1-t)^{n}}{(t ; t)_{n}} \sum_{\lambda_{1}, \ldots, \lambda_{n}=-\infty}^{\infty} h_{\lambda} \prod_{1 \leq i<j \leq n} \frac{q^{\lambda_{i}}-t q^{\lambda_{j}}}{q^{\lambda_{i}}-q^{\lambda_{j}}} .
$$

Note that because of the symmetry $3.5 \mathrm{a}$ not all of the inequalities in $3.5 \mathrm{~b}$ ) are independent. For example, $3.5 \mathrm{~b}$ follows from the weaker $h_{\lambda}=0$ if $\lambda_{i}-\lambda_{i+1} \in$ $\{0, \ldots, k-1\}$ for all $i \in\{1, \ldots, n-1\}$.

Before giving a proof, let us first show how Lemma 3.1 can be applied to transform (3.4) into the statement of Theorem 1.1. To this end we identify $h_{\lambda+k \delta}$ with the summand on the left of 3.4 . Hence, by $|\delta|=\left(\begin{array}{l}n \\ 2\end{array}\right), n(\delta)=\left(\begin{array}{l}n \\ 3\end{array}\right)$ and $n(\lambda)=\sum_{i<j} \lambda_{j}$,

$$
\begin{aligned}
h_{\lambda}=q^{-\alpha k\left(\begin{array}{c}
n \\
2
\end{array}\right)-2 k^{2}\left(\begin{array}{l}
n \\
3
\end{array}\right)+\alpha|\lambda|} \frac{P_{\mu}\left(q^{\lambda}\right)}{P_{\mu}\left(q^{k \delta}\right)} \prod_{i=1}^{n} \frac{\left(-c q^{\alpha+\beta+2(n-1) k+\lambda_{i}} ; q\right)_{\infty}}{\left(-c q^{\lambda_{i}} ; q\right)_{\infty}} \\
\quad \times \prod_{1 \leq i<j \leq n} q^{(2 k-1) \lambda_{j}}\left(q^{\lambda_{j}}-q^{\lambda_{i}}\right)\left(q^{1-k+\lambda_{i}-\lambda_{j}} ; q\right)_{2 k-1} .
\end{aligned}
$$

Now clearly the first line on the right is symmetric in $\lambda$. To see that also the second line is symmetric note that by

$$
(a ; q)_{n}=\left(q^{1-n} / a ; q\right)_{n}(-a)^{n} q^{\left(\begin{array}{c}
n \\
2
\end{array}\right)}
$$

both $\prod_{i<j}\left(q^{\lambda_{j}}-q^{\lambda_{i}}\right)$ and $\prod_{i<j} q^{(2 k-1) \lambda_{j}}\left(q^{1-k+\lambda_{i}-\lambda_{j}} ; q\right)_{2 k-1}$ are skew-symmetric functions (of $\lambda$ ), making their product a symmetric function. Next observe that thanks to the factor $\left(q^{1-k+\lambda_{i}-\lambda_{j}} ; q\right)_{2 k-1}$ also the inequalities $(3.5 \mathrm{~b})$ hold, putting us in a position to apply Lemma 3.1. Choosing $t=q^{k}$ in the lemma, recalling definition (1.4), and using the above-discussed fact that the second line in the expression for $h_{\lambda}$ is symmetric in $\lambda$, so that we may interchange $\lambda_{i}$ and $\lambda_{j}$, we obtain the second integral evaluation of Theorem 1.1 with $\lambda$ replaced by $\mu$.

The conditions on $\alpha$ and $\beta$ imposed by the theorem follow from the fact that, taking into account all the variable changes, we have effectively applied (2.17) with $x_{i} \mapsto q^{\alpha+(n-i) k+\lambda_{i}}$ and $b / a \mapsto q^{\alpha+\beta+2(n-1) k}$. Since $0<q<1$ the inequality $\left|b t^{1-n} / a\right|<\left|x_{i}\right|<1$ (with $t=q^{k}$ ) therefore translates into $\operatorname{Re}(\alpha+\beta)+(n-1) k>$ $\operatorname{Re}(\alpha)+(n-i) k+\lambda_{i}>0$. Since $\lambda$ is a partition this yields $\operatorname{Re}(\alpha)>-\lambda_{n}$ and $\operatorname{Re}(\beta)>\lambda_{1}$.

Proof of Lemma 3.1. We take the left-hand side of the identity of the lemma and replace $\lambda \mapsto \lambda-k \delta$, i.e., $\lambda_{i} \mapsto \lambda_{i}-k(n-i)$. This leads to the sum $\sum_{\lambda \in P}^{\prime} h_{\lambda}$ where the prime denotes the added restriction $\lambda_{i}-\lambda_{i+1} \geq k$. Since $h_{\lambda}=0$ for $\lambda_{i}-\lambda_{i+1} \in\{0, \ldots, k-1\}$ we may drop the prime to simply get $\sum_{\lambda \in P} h_{\lambda}$. Now by the symmetry $3.5 \mathrm{a}$ and the fact that $h_{\lambda}=0$ for $\lambda_{i}=\lambda_{j}$ we may further replace this by $(1 / n !) \sum_{\lambda_{1}, \ldots, \lambda_{n}=-\infty}^{\infty} h_{\lambda}$. Next we need the summation identity [20, Equation (III.1.4)]

$$
\sum_{w \in S_{n}} w\left(\prod_{1 \leq i<j \leq n} \frac{x_{i}-t x_{j}}{x_{i}-x_{j}}\right)=\prod_{i=1}^{n} \frac{1-t^{i}}{1-t} .
$$

(This follows by viewing the symmetric function on the left as the ratio of two skew-symmetric polynomials - the denominator polynomial being the Vandermonde 
determinant. Since both polynomials are of degree $n-1$ and vanish for $x_{i}=x_{j}$, the left must be independent of $x$ and may be evaluated by taking $x=t^{\delta}$. For this specialization the only non-vanishing contribution comes from the (unique) permutation of maximum length, leading to the desired right-side.) Hence

$$
\begin{aligned}
\sum_{\lambda_{1}, \ldots, \lambda_{n}=-\infty}^{\infty} h_{\lambda} & =\frac{(1-t)^{n}}{(t ; t)_{n}} \sum_{\lambda_{1}, \ldots, \lambda_{n}=-\infty}^{\infty} h_{\lambda} \sum_{w \in S_{n}} w\left(\prod_{1 \leq i<j \leq n} \frac{q^{\lambda_{i}}-t q^{\lambda_{j}}}{q^{\lambda_{i}}-q^{\lambda_{j}}}\right) \\
& =\frac{(1-t)^{n}}{(t ; t)_{n}} \sum_{\lambda_{1}, \ldots, \lambda_{n}=-\infty}^{\infty} \sum_{w \in S_{n}} w\left(h_{\lambda} \prod_{1 \leq i<j \leq n} \frac{q^{\lambda_{i}}-t q^{\lambda_{j}}}{q^{\lambda_{i}}-q^{\lambda_{j}}}\right) \\
& =n ! \frac{(1-t)^{n}}{(t ; t)_{n}} \sum_{\lambda_{1}, \ldots, \lambda_{n}=-\infty}^{\infty} h_{\lambda} \prod_{1 \leq i<j \leq n} \frac{q^{\lambda_{i}}-t q^{\lambda_{j}}}{q^{\lambda_{i}}-q^{\lambda_{j}}} .
\end{aligned}
$$

3.2. Proof of Theorem 1.4. Let the structure constants $f_{\lambda \mu}^{\nu}=f_{\lambda \mu}^{\nu}(q, t)$ be given by [20, Equation (VI.7.1')]

$$
P_{\lambda}(x) P_{\mu}(x)=\sum_{\nu} f_{\lambda \mu}^{\nu} P_{\nu}(x)
$$

Substituting this in the left-hand side of $(1.9)$ and performing the resulting integral by the $\beta=k$ case of Corollary 1.3 we get

$$
\begin{aligned}
\operatorname{LHS} 1.9]=q^{\alpha k\left(\begin{array}{c}
n \\
2
\end{array}\right)+} & 2 k^{2}\left(\begin{array}{c}
n \\
3
\end{array}\right)(1-q)^{k n^{2}} \prod_{i=1}^{n} \frac{\Gamma_{q}(i k) \Gamma_{q}(i k+1)}{\Gamma_{q}(k+1)} \\
& \times \sum_{\nu} f_{\lambda \mu}^{\nu}\left(q, q^{k}\right) P_{\nu}\left(q^{k \delta} ; q, q^{k}\right) \prod_{i=1}^{n} \frac{1}{\left(q^{\alpha+(n-i) k+\nu_{i}} ; q\right)_{n k}}
\end{aligned}
$$

where we have used

$$
\frac{\Gamma_{q}(a+n)}{\Gamma_{q}(a)}=\frac{\left(q^{a} ; q\right)_{n}}{(1-q)^{n}} .
$$

To perform the sum over $\nu$ we need the following proposition.

Proposition 3.1. Let $\lambda=\left(\lambda_{1}, \ldots, \lambda_{n}\right)$ and $\mu=\left(\mu_{1}, \ldots, \mu_{n}\right)$ be partitions. Then

$$
\sum_{\nu} f_{\lambda \mu}^{\nu} P_{\nu}\left(t^{\delta}\right) \frac{\left(z t^{-n}\right)_{\nu}}{(z)_{\nu}}=P_{\lambda}\left(t^{\delta}\right) P_{\mu}\left(t^{\delta}\right) \prod_{i, j=1}^{n} \frac{\left(z t^{1-i-j} ; q\right)_{\lambda_{i}+\mu_{j}}}{\left(z t^{2-i-j} ; q\right)_{\lambda_{i}+\mu_{j}}} .
$$

Note that for given $\lambda$ and $\mu$ this result is a rational function identity. Indeed, by the homogeneity of the Macdonald polynomials, $f_{\lambda \mu}^{\nu}=0$ if $|\nu| \neq|\lambda|+|\mu|$, so that only a finite number of terms contribute to the sum on the left. In view of this it clearly suffices to prove the proposition for $|z|<\min \left\{\left|t^{n+i-1}\right|\right\}_{i=1}^{n}$ (i.e., $|z|<|t|^{n}$ if $|t| \geq 1$ and $|z|<|t|^{2 n-1}$ if $\left.|t| \leq 1\right)$. This restriction on $z$ in the proof given below arises from the use of the $q$-binomial theorem (2.17).

Proof of Proposition 3.1. By 2.19) we first rewrite the above identity as

$$
\sum_{\nu} f_{\lambda \mu}^{\nu} P_{\nu}\left(t^{\delta}\right) \prod_{i=1}^{n} \frac{\left(z t^{1-i} q^{\nu_{i}} ; q\right)_{\infty}}{\left(z t^{1-n-i} q^{\nu_{i}} ; q\right)_{\infty}}=P_{\lambda}\left(t^{\delta}\right) P_{\mu}\left(t^{\delta}\right) \prod_{i, j=1}^{n} \frac{\left(z t^{2-i-j} q^{\lambda_{i}+\mu_{j}} ; q\right)_{\infty}}{\left(z t^{1-i-j} q^{\lambda_{i}+\mu_{j}} ; q\right)_{\infty}}
$$


For the sake of compactness of subsequent equations we set $z=y t^{2 n-1}$ and then use the ${ }_{1} \Phi_{0}$ sum 2.17) to expand the product over $i$ in the summand on the left. Hence

$$
\operatorname{LHS} 3.12=\sum_{\nu, \eta} f_{\lambda \mu}^{\nu} \frac{\left(t^{n}\right)_{\eta} y^{|\eta|}}{c_{\eta}^{\prime}} u_{\nu}\left(P_{\eta}\right) u_{0}\left(P_{\nu}\right)
$$

where we have used 2.5 and $P_{\nu}\left(t^{\delta}\right)=u_{0}\left(P_{\nu}\right)$. By the symmetry 2.15 this yields

$$
\operatorname{LHS} 3.12=\sum_{\nu, \eta} f_{\lambda \mu}^{\nu} \frac{\left(t^{n}\right)_{\eta} y^{|\eta|}}{c_{\eta}^{\prime}} u_{\eta}\left(P_{\nu}\right) u_{0}\left(P_{\eta}\right)
$$

The sum over $\nu$ can now be carried out by $(3.9)$, leading to

$$
\text { LHS } 3.12=\sum_{\eta} \frac{\left(t^{n}\right)_{\eta} y^{|\eta|}}{c_{\eta}^{\prime}} u_{\eta}\left(P_{\lambda}\right) u_{\eta}\left(P_{\mu}\right) u_{0}\left(P_{\eta}\right) .
$$

By two more applications of 2.15 this gives

$$
\text { LHS 3.12 }=u_{0}\left(P_{\lambda}\right) u_{0}\left(P_{\mu}\right) \sum_{\eta} \frac{\left(t^{n}\right)_{\eta} y^{|\eta|}}{c_{\eta}^{\prime}} \frac{u_{\lambda}\left(P_{\eta}\right) u_{\mu}\left(P_{\eta}\right)}{u_{0}\left(P_{\eta}\right)} \text {. }
$$

From $(2.8)$ and $(2.14 \mathrm{a})$ it thus follows that

$$
\operatorname{LHS} 3.12=u_{0}\left(P_{\lambda}\right) u_{0}\left(P_{\mu}\right) \sum_{\eta} b_{\eta} y^{|\eta|} u_{\lambda}\left(P_{\eta}\right) u_{\mu}\left(P_{\eta}\right) .
$$

This nearly completes the proof. All that is needed now is the homogeneity of the Macdonald polynomials and the Cauchy identity (2.11). By virtue of these,

$$
\operatorname{LHS} 3.12=u_{0}\left(P_{\lambda}\right) u_{0}\left(P_{\mu}\right) \prod_{i, j=1}^{n} \frac{\left(y t^{2 n+1-i-j} q^{\lambda_{i}+\mu_{j}} ; q\right)_{\infty}}{\left(y t^{2 n-i-j} q^{\lambda_{i}+\mu_{j}} ; q\right)_{\infty}} .
$$

Recalling that $z=y q^{2 n-1}$ this is in accordance with the right-hand side of 3.12 .

We remark here that a dual version of Proposition 3.1 without the free parameter $z$ is given by Proposition 3.2 of Section 3.6 .

We now return to the proof of Theorem 1.4 Replacing $z \mapsto q^{\alpha} t^{2 n-1}$ and then choosing $t=q^{k}$, the identity of Proposition 3.1 can be written as

$$
\begin{aligned}
\sum_{\nu} f_{\lambda \mu}^{\nu}\left(q, q^{k}\right) P_{\nu}\left(q^{k \delta} ; q, q^{k}\right) \prod_{i=1}^{n} \frac{1}{\left(q^{\alpha+(n-i) k+\nu_{i}} ; q\right)_{n k}} \\
\quad=P_{\lambda}\left(q^{k \delta} ; q, q^{k}\right) P_{\mu}\left(q^{k \delta} ; q, q^{k}\right) \prod_{i, j=1}^{n} \frac{1}{\left(q^{\alpha+(2 n-i-j) k+\lambda_{i}+\mu_{j}} ; q\right)_{k}} .
\end{aligned}
$$

Using this to perform the sum over $\nu$ in 3.10 results in the right-hand side of (1.9).

In the above we have not yet addressed to condition $\operatorname{Re}(\alpha)>-\lambda_{n}-\mu_{n}$ imposed on the theorem. To see how this arises note that we first applied Corollary 1.3 with $\beta=k$ and $\lambda \mapsto \nu$. This step is justified if $\operatorname{Re}(\alpha)>-\nu_{n}$. The next step was to perform the sum over $\nu$ in 3.10. According to [20, Equation (7.4)] $f_{\lambda \mu}^{\nu}=0$ unless $\nu \supset \lambda$ and $\nu \supset \mu$. Hence we may without loss of generality assume that $\nu_{n} \geq \mu_{n}$. Recalling the notation (2.1), let $\tilde{\mu}$ and $\tilde{\nu}$ be the partitions $\tilde{\mu}=\mu-\mu_{n}$ and $\tilde{\nu}=\nu-\mu_{n}$. From (3.9) and (1.8) we have $P_{\lambda} P_{\tilde{\mu}}=\sum_{\nu} f_{\lambda \mu}^{\nu} P_{\tilde{\nu}}$. Since also 
$P_{\lambda} P_{\tilde{\mu}}=\sum_{\tilde{\nu}} f_{\lambda \tilde{\mu}}^{\tilde{\nu}} P_{\tilde{\nu}}$ we conclude that $f_{\lambda \mu}^{\nu}=f_{\lambda \tilde{\mu}}^{\tilde{\nu}}$. But $f_{\lambda \tilde{\mu}}^{\tilde{\nu}}=0$ if $\tilde{\nu} \not \supset \lambda$. Hence $f_{\lambda \mu}^{\nu}=0$ if $\nu_{n}<\lambda_{n}+\mu_{n}$. This last result implies that by summing over $\nu$ in (3.10) the condition $\operatorname{Re}(\alpha)>-\nu_{n}$ on the summand translates into $\operatorname{Re}(\alpha)>-\lambda_{n}-\mu_{n}$.

3.3. Proof of Corollary 1.6. To obtain this from Theorem 1.4 is rather straightforward. Multiplying boths sides of $(1.9)$ by $(-1)^{|\mu|} P_{\mu^{\prime}}\left(y ; q^{k}, q\right)$ and summing over $\mu$ such that $\mu_{1}=\ell\left(\mu^{\prime}\right) \leq m$ we find by the use of the Cauchy identity 2.12 that

$$
\begin{aligned}
\text { LHS 1.10 } & =q^{\alpha k\left(\begin{array}{c}
n \\
2
\end{array}\right)+2 k^{2}\left(\begin{array}{c}
n \\
3
\end{array}\right)(1-q)^{k n^{2}} P_{\lambda}\left(q^{k \delta} ; q, q^{k}\right)} \prod_{i=1}^{n} \frac{\Gamma_{q}(i k) \Gamma_{q}(i k+1)}{\Gamma_{q}(k+1)} \\
\times & \sum_{\substack{\mu \\
\mu_{1} \leq m}}(-1)^{|\mu|} P_{\mu^{\prime}}\left(y ; q^{k}, q\right) P_{\mu}\left(q^{k \delta} ; q, q^{k}\right) \prod_{i, j=1}^{n} \frac{1}{\left(q^{\alpha+(2 n-i-j) k+\lambda_{i}+\mu_{j}} ; q\right)_{k}} .
\end{aligned}
$$

Next we eliminate $P_{\mu}\left(q^{k \delta} ; q, q^{k}\right)$ by 2.14a), use

$$
\begin{aligned}
& \prod_{i, j=1}^{n} \frac{1}{\left(q^{\alpha+(2 n-i-j) k+\lambda_{i}+\mu_{j}} ; q\right)_{k}} \\
& \quad=\frac{1}{(1-q)^{k n^{2}}} \prod_{i=1}^{n} \frac{\Gamma_{q}\left(\alpha+(n-i) k+\lambda_{i}\right)}{\Gamma_{q}\left(\alpha+(2 n-i) k+\lambda_{i}\right)} \frac{\left(q^{\alpha+(2 n-i-1) k+\lambda_{i}} ; q, q^{k}\right)_{\mu}}{\left(q^{\alpha+(2 n-i) k+\lambda_{i}} ; q, q^{k}\right)_{\mu}}
\end{aligned}
$$

as follows from

$$
\left(a q^{n} ; q\right)_{k}=\frac{(a ; q)_{k}\left(a q^{k} ; q\right)_{n}}{(a ; q)_{n}}
$$

2.9) and (3.11), and replace the summation index $\mu$ by $\mu^{\prime}$. As a result

LHS 1.10

$$
\begin{aligned}
& =q^{\alpha k\left(\begin{array}{c}
n \\
2
\end{array}\right)+2 k^{2}\left(\begin{array}{c}
n \\
3
\end{array}\right)} P_{\lambda}\left(q^{k \delta} ; q, q^{k}\right) \prod_{i=1}^{n} \frac{\Gamma_{q}\left(\alpha+(n-i) k+\lambda_{i}\right) \Gamma_{q}(i k) \Gamma_{q}(i k+1)}{\Gamma_{q}\left(\alpha+(2 n-i) k+\lambda_{i}\right) \Gamma_{q}(k+1)} \\
& \quad \times \sum_{\substack{\mu \\
\mu_{1} \leq n}}(-1)^{|\mu|} \frac{\left(q^{n k} ; q, q^{k}\right)_{\mu^{\prime}} P_{\mu}\left(y ; q^{k}, q\right)}{c_{\mu^{\prime}}\left(q, q^{k}\right)} \prod_{i=1}^{n} \frac{\left(q^{\alpha+(2 n-i-1) k+\lambda_{i}} ; q, q^{k}\right)_{\mu^{\prime}}}{\left(q^{\alpha+(2 n-i) k+\lambda_{i}} ; q, q^{k}\right)_{\mu^{\prime}}}
\end{aligned}
$$

with $\mu=\left(\mu_{1}, \ldots, \mu_{m}\right)$. Comparing this with the right-hand side of 1.10$)$ it remains to show that the last line can be written as the ${ }_{n+1} \Phi_{n}$ series claimed in the corollary. For this we need

$$
(a ; q, t)_{\lambda^{\prime}}=(-1)^{|\lambda|}\left(a^{-1} ; t, q\right)_{\lambda} \quad \text { and } \quad c_{\lambda^{\prime}}(q, t)=c_{\lambda}^{\prime}(t, q)
$$

both of which follow immediately from the definitions of the respective functions in terms of the arm-(co)length and leg-(co)length and the fact that under transposition arms become legs and and legs become arms. The sum over $\mu$ may thus be written as

$$
\sum_{\substack{\mu \\ \mu_{1} \leq n}} \frac{\left(q^{-n k} ; q^{k}, q\right)_{\mu} P_{\mu}\left(y ; q^{k}, q\right)}{c_{\mu}^{\prime}\left(q^{k}, q\right)} \prod_{i=1}^{n} \frac{\left(q^{-\alpha-(2 n-i-1) k-\lambda_{i}} ; q^{k}, q\right)_{\mu}}{\left(q^{-\alpha-(2 n-i) k-\lambda_{i}} ; q^{k}, q\right)_{\mu}} .
$$

By definition (2.16) this is seen to be in accordance with the ${ }_{n+1} \Phi_{n}$ series on the right of 1.10. 
3.4. Proof of Theorem 1.7. The proof presented below is a streamlined $q$-version of the proof of Corollary 1.8 given by Kadell [13.

First we show that it suffices to proof the theorem for $a=0$, or, equivalently, for $a=k-1$. To this end we assume $a>0$ and replace $\mu \mapsto \mu+a$. Next we twice apply (2.6) (with $\lambda \mapsto \mu$ ) and use both (3.2) and (3.7) to write

$$
\left(x_{i} ; q\right)_{a}\left(q / x_{i} ; q\right)_{k-a-1}=\left(-x_{i}\right)^{a} q^{\left(\begin{array}{c}
a \\
2
\end{array}\right)}\left(q^{1-a} / x_{i} ; q\right)_{k-1} .
$$

Comparing the resulting constant term identity with Theorem 1.7 for $a=0$, we conclude that the $a>0$ cases follow from the $a=0$ case if

$$
\begin{aligned}
& \text { С.T. }\left[P_{\lambda}\left(x ; q, q^{k}\right) P_{\mu}\left(x^{-1} ; q, q^{k}\right) \prod_{i=1}^{n}\left(\frac{q^{1-a}}{x_{i}} ; q\right)_{k-1} \prod_{1 \leq i<j \leq n}\left(\frac{x_{i}}{x_{j}}, \frac{q x_{j}}{x_{i}} ; q\right)_{k}\right] \\
& =g_{\lambda, \mu}^{a}(q) \text { C.T. }\left[P_{\lambda}\left(x ; q, q^{k}\right) P_{\mu}\left(x^{-1} ; q, q^{k}\right) \prod_{i=1}^{n}\left(\frac{q}{x_{i}} ; q\right)_{k-1} \prod_{1 \leq i<j \leq n}\left(\frac{x_{i}}{x_{j}}, \frac{q x_{j}}{x_{i}} ; q\right)_{k}\right] .
\end{aligned}
$$

with $g_{\lambda, \mu}^{a}(q)=q^{a(|\mu|-|\lambda|)}$. This is readily established by the substitution $x_{i} \mapsto x_{i} q^{-a}$ on the left. Although this changes the expression within the square brackets, it does not alter the constant term. The homogeneity 2.5 of the Macdonald polynomials now does the rest.

Below we present a proof for $a=k-1$. By the comment following Theorem 1.7 this is equivalent to proving the case $a=0$.

Let us denote the $a=k-1$ instance of the expression within the square brackets in Theorem 1.7 by $S_{\lambda, \mu}^{k}(q)$. For $N$ a fixed integer such that $\mu_{1} \leq N$ denote $\hat{\mu}$ the complement of $\mu$ with respect to $\left(N^{n}\right)$, i.e., $\hat{\mu}_{i}=N-\mu_{n-i+1}$ for $i \in\{1, \ldots, n\}$. Then, by

$$
P_{\mu}(x ; q, t)=\left(x_{1} \cdots x_{n}\right)^{N} P_{\hat{\mu}}\left(x^{-1} ; q, t\right)
$$

(see e.g., [6]), 3.7 and (3.2),

$$
\begin{aligned}
S_{\lambda, \mu}^{k}(q)=(-1)^{k\left(\begin{array}{c}
n \\
2
\end{array}\right)} q^{\left(\begin{array}{c}
n \\
2
\end{array}\right)\left(\begin{array}{l}
k \\
2
\end{array}\right)} P_{\lambda}\left(x ; q, q^{k}\right) P_{\hat{\mu}}\left(x ; q, q^{k}\right) & \\
& \times \prod_{i=1}^{n} x_{i}^{-(n-1) k-N}\left(x_{i} ; q\right)_{k-1} \prod_{1 \leq i<j \leq n} x_{i}^{2 k}\left(\frac{x_{j} q^{1-k}}{x_{i}} ; q\right)_{2 k}
\end{aligned}
$$

Since we need the constant term of $S_{\lambda, \mu}^{k}(q)$ we may replace $x \mapsto q x$ and use the homogeneity of the Macdonald polynomials and $n N-|\hat{\mu}|=|\mu|$ to get

$$
\begin{aligned}
\text { C.T. }\left[S_{\lambda, \mu}^{k}(q)\right]= & (-1)^{k\left(\begin{array}{c}
n \\
2
\end{array}\right)} q^{\left(\begin{array}{c}
n \\
2
\end{array}\right)\left(\begin{array}{l}
k \\
2
\end{array}\right)+|\lambda|-|\mu|} \text { C.T. }\left[P_{\lambda}\left(x ; q, q^{k}\right) P_{\hat{\mu}}\left(x ; q, q^{k}\right)\right. \\
& \left.\times \prod_{i=1}^{n} x_{i}^{-(n-1) k-N}\left(x_{i} q ; q\right)_{k-1} \prod_{1 \leq i<j \leq n} x_{i}^{2 k}\left(\frac{x_{j} q^{1-k}}{x_{i}} ; q\right)_{2 k}\right] .
\end{aligned}
$$

Our next step is to use the well-known and easy to prove fact (see e.g., [3, 9, 17]) that if $f(x)$ is a Laurent polynomial in $x$, then

$$
\text { C.T. }[f(x)]=\lim _{\varepsilon \rightarrow 0}\left(\frac{1-q^{\varepsilon}}{1-q}\right)^{n} \int_{[0,1]^{n}}\left(\prod_{i=1}^{n} x_{i}^{\varepsilon-1}\right) f(x) \mathrm{d}_{q} x .
$$


According to the above we may compute the right-hand side of 3.15 by the $\alpha \mapsto$ $\varepsilon-N-(n-1) k$ and $\mu \mapsto \hat{\mu}$ instance of Theorem 1.4 (where the integral in 1.9 ) is analytically continued using its evaluation). Replacing $q^{\varepsilon}$ by $\omega$ this yields

$$
\begin{aligned}
& \text { C.T. }\left[S_{\lambda, \mu}^{k}(q)\right]=(-1)^{k\left(\begin{array}{c}
n \\
2
\end{array}\right)} q^{\left(\begin{array}{c}
n \\
2
\end{array}\right)\left(\begin{array}{c}
k \\
2
\end{array}\right)-k^{2}\left(\begin{array}{c}
n+1 \\
3
\end{array}\right)+|\lambda|-|\mu|-N k\left(\begin{array}{c}
n \\
2
\end{array}\right)} \\
& \times P_{\lambda}\left(q^{k \delta} ; q, q^{k}\right) P_{\hat{\mu}}\left(q^{k \delta} ; q, q^{k}\right) \prod_{i=1}^{n} \frac{(q ; q)_{i k-1}(q ; q)_{i k}}{(q ; q)_{k}} \\
& \times \lim _{\omega \rightarrow 1}(1-\omega)^{n} \prod_{i, j=1}^{n} \frac{1}{\left(\omega q^{(n-i-j+1) k-N+\lambda_{i}+\hat{\mu}_{j}} ; q\right)_{k}},
\end{aligned}
$$

where we have also used 1.2 to eliminate some $q$-gamma functions. We now eliminate $\hat{\mu}$ in favour of $\mu$. To achieve this we replace $j \mapsto n+1-j$ in the double product on the right and use 2.14b to obtain

$$
P_{\hat{\mu}}\left(q^{\delta}\right)=t^{N\left(\begin{array}{l}
n \\
2
\end{array}\right)-(n-1)|\mu|} P_{\mu}\left(q^{\delta}\right) .
$$

Hence

$$
\begin{aligned}
& \text { C.T. }\left[S_{\lambda, \mu}^{k}(q)\right]=(-1)^{k}\left(\begin{array}{c}
n \\
2
\end{array}\right) q^{\left(\begin{array}{c}
n \\
2
\end{array}\right)\left(\begin{array}{c}
k \\
2
\end{array}\right)-k^{2}\left(\begin{array}{c}
n+1 \\
3
\end{array}\right)+|\lambda|-|\mu|-k(n-1)|\mu|} \\
& \quad \times P_{\lambda}\left(q^{k \delta} ; q, q^{k}\right) P_{\mu}\left(q^{k \delta} ; q, q^{k}\right) \prod_{i=1}^{n} \frac{(q ; q)_{i k-1}(q ; q)_{i k}}{(q ; q)_{k}} \\
& \quad \times \lim _{\omega \rightarrow 1}(1-\omega)^{n} \prod_{i, j=1}^{n} \frac{1}{\left(\omega q^{(j-i) k+\lambda_{i}-\mu_{j}} ; q\right)_{k}} .
\end{aligned}
$$

It remains to deal with the limit of the double product. For fixed $i$ consider

$$
\prod_{j=1}^{n} \frac{1}{\left(\omega q^{(j-i) k+\lambda_{i}-\mu_{j}} ; q\right)_{k}} \text {. }
$$

It has a pole at $\omega=1$ if there is a $j \in\{1, \ldots, n\}$ such that $(j-i) k+\lambda_{i}-\mu_{j} \in$ $\{1-k, \ldots, 0\}$. Clearly, there can at most be one such $j$, since

$$
\left((j+1-i) k+\lambda_{i}-\mu_{j+1}\right)-\left((j-i) k+\lambda_{i}-\mu_{j}\right)=k+\mu_{j}-\mu_{j+1} \geq k .
$$

Hence (3.17) has at most one simple pole at $\omega=1$. Since $(1-\omega)^{n}$ has a zero of order $n$ at $\omega=1$ it follows that the C.T. $\left[S_{\lambda, \mu}^{k}(q)\right]=0$ unless for each $i \in\{1, \ldots, n\}$ the product (3.17) has a pole. Hence for the constant term to be non-zero there must for each $i$ be a (unique) $j$, say $j_{i}$, such that

$$
\left(j_{i}-i\right) k+\lambda_{i}-\mu_{j_{i}} \in\{1-k, \ldots, 0\} .
$$

Therefore

$$
\left(\left(j_{i}-i\right) k+\lambda_{i}-\mu_{j_{i}}\right)-\left(\left(j_{i+1}-i-1\right) k+\lambda_{i+1}-\mu_{j_{i+1}}\right) \in\{1-k, \ldots, k-1\} .
$$

Since $\lambda_{i}-\lambda_{i+1} \geq 0$ this implies that $\left(j_{i}-j_{i+1}\right) k+\mu_{j_{i+1}}-\mu_{j_{i}}<0$. If $j_{i} \geq j_{i+1}$ then $\mu_{j_{i+1}}-\mu_{j_{i}} \geq 0$ so that $\left(j_{i}-j_{i+1}\right) k+\mu_{j_{i+1}}-\mu_{j_{i}} \geq 0$. Since this would lead to a contradiction, we infer that $j_{i}<j_{i+1}$ for all $i$, which implies that $j_{i}=i$. Substituting this back into (3.18) we conclude that C.T. $\left[S_{\lambda, \mu}^{k}(q)\right]=0$ unless

$$
\mu_{i}-\lambda_{i} \in\{0, \ldots, k-1\} \text { for all } i \in\{1, \ldots, n\} .
$$

This is in accordance with the comments made following Theorem 1.7 
In the remainder we assume $\sqrt{3.19}$ to hold. Then

$$
\begin{aligned}
& \lim _{\omega \rightarrow 1}(1-\omega)^{n} \prod_{i, j=1}^{n} \frac{1}{\left(\omega q^{(j-i) k+\lambda_{i}-\mu_{j}} ; q\right)_{k}} \\
& =\prod_{\substack{i, j=1 \\
i \neq j}}^{n} \frac{1}{\left(q^{(j-i) k+\lambda_{i}-\mu_{j}} ; q\right)_{k}} \lim _{\omega \rightarrow 1} \prod_{i=1}^{n} \frac{1-\omega}{\left(\omega q^{\lambda_{i}-\mu_{i}} ; q\right)_{k}} \\
& \left.=\frac{(-1)^{|\lambda|+|\mu|}}{(q ; q)_{k-1}^{n}} \prod_{i=1}^{n} q^{\left(\lambda_{i}-\mu_{i}\right.}\right)\left[\begin{array}{c}
k-1 \\
\mu_{i}-\lambda_{i}
\end{array}\right] \prod_{\substack{i, j=1 \\
i \neq j}}^{n} \frac{1}{\left(q^{(j-i) k+\lambda_{i}-\mu_{j}} ; q\right)_{k}} \\
& =(-1)^{|\lambda|+|\mu|+k\left(\begin{array}{c}
n \\
2
\end{array}\right)} q^{k^{2}\left(\begin{array}{c}
n+1 \\
3
\end{array}\right)-\left(\begin{array}{c}
n \\
2
\end{array}\right)\left(\begin{array}{l}
k \\
2
\end{array}\right)-k\{n(\lambda)+n(\mu)-(n-1)|\mu|\}} \\
& \left.\times \frac{1}{(q ; q)_{k-1}^{n}} \prod_{i=1}^{n} q^{\left(\lambda_{i}-\mu_{i}\right.}\right)\left[\begin{array}{c}
k-1 \\
\mu_{i}-\lambda_{i}
\end{array}\right]_{q} \\
& \times \prod_{1 \leq i<j \leq n} \frac{1}{\left(q^{(j-i) k+\lambda_{i}-\mu_{j}} ; q\right)_{k}\left(q^{1-k+(j-i) k+\mu_{i}-\lambda_{j}} ; q\right)_{k}} .
\end{aligned}
$$

where we have used the easily proved

$$
\lim _{\omega \rightarrow 1} \frac{1-\omega}{\left(\omega q^{-a} ; q\right)_{k}}=\frac{(-1)^{a} q^{\left(\begin{array}{c}
a+1 \\
2
\end{array}\right)}}{(q ; q)_{k-1}}\left[\begin{array}{c}
k-1 \\
a
\end{array}\right]_{q}
$$

and (3.7). Substituting the above in 3.16 and using (1.7) to eliminate the specialized Macdonald polynomials completes the proof of the $a=k-1$ instance of Theorem 1.7.

3.5. Proof of Corollary 1.9. We first invoke the following simple lemma, which is essentially the content of parts (a)-(c) of [20, Example VI.9.1], to show that Theorem 1.7 is equivalent to 1.13 .

Lemma 3.2. Let $f(x)$ be a symmetric Laurent polynomial. Then

$$
\text { C.T. }\left[f(x) \prod_{1 \leq i<j \leq n}\left(\frac{x_{i}}{x_{j}}, \frac{x_{j}}{x_{i}} ; q\right)_{k}\right]=n ! \frac{\left(1-q^{k}\right)^{n}}{\left(q^{k} ; q^{k}\right)_{n}} \text { C.T. }\left[f(x) \prod_{1 \leq i<j \leq n}\left(\frac{x_{i}}{x_{j}}, \frac{q x_{j}}{x_{i}} ; q\right)_{k}\right] .
$$

Since $P_{\lambda}(x) P_{\mu}\left(x^{-1}\right) h_{a, k}(x)$ with $h_{a, k}$ given by 11.14$)$ is a symmetric Laurent polynomial this clearly establishes the equivalence of Theorem 1.7 and the scalar product evaluation of 1.13 .

Proof of Lemma 3.2. Suppressing their $k$-dependence, let us denote the expressions within the square brackets on the left and right by $L(x)$ and $R(x)$, respectively. Clearly, $L(x)=w(L(x))$, C.T. $[R(x)]=$ C.T. $[w(R(x))]$ and

$$
R(x)=L(x) \prod_{1 \leq i<j \leq n} \frac{x_{i}-q^{k} x_{j}}{x_{i}-x_{j}} .
$$


Combining these facts gives

$$
\begin{aligned}
\text { C.T. }[R(x)] & =\frac{1}{n !} \sum_{w \in S_{n}} \text { C.T. }[w(R(x))] \\
& =\frac{1}{n !} \text { C.T. }\left[L(x) \sum_{w \in S_{n}} w\left(\prod_{1 \leq i<j \leq n} \frac{x_{i}-q^{k} x_{j}}{x_{i}-x_{j}}\right)\right] \\
& =\frac{1}{n !} \frac{\left(q^{k} ; q^{k}\right)_{n}}{\left(1-q^{k}\right)^{n}} \text { C.T. }[L(x)],
\end{aligned}
$$

where in the final step we have used $(3.8)$.

Next we show that 1.13$)$ implies 1.15$)$. Since $P_{\lambda}(x) P_{\mu}\left(x^{-1}\right)$ is homogeneous of degree $|\lambda|-|\mu|, \prod_{i<j}\left(x_{i} / x_{j}, x_{j} / x_{i} ; q\right)_{k}$ is homogeneous of degree zero and $h_{k-1, k}(x)$ is a polynomial with constant term 1 , it follows that

$$
\left\langle P_{\lambda}, P_{\mu}\right\rangle^{\prime}=\left\langle P_{\lambda}, P_{\mu}\right\rangle^{\prime} \delta_{|\lambda|,|\mu|}=\left\langle P_{\lambda} h_{k-1, k}, P_{\mu}\right\rangle^{\prime} \delta_{|\lambda|,|\mu|}
$$

From (1.13) we know that $\left\langle P_{\lambda} h_{k-1, k}, P_{\mu}\right\rangle^{\prime}=0$ unless $\mu_{i}-\lambda_{i} \in\{0, \ldots, k-1\}$ for all $i \in\{1, \ldots, n\}$. Combined with the fact that $|\lambda|=|\mu|$ this yields that $\lambda=\mu$. Hence

$$
\left\langle P_{\lambda}, P_{\mu}\right\rangle^{\prime}=\left\langle P_{\lambda} h_{k-1, k}, P_{\mu}\right\rangle^{\prime} \delta_{\lambda \mu} .
$$

Using 1.13 with $\lambda=\mu$ and $a=k-1$ to evaluate the scalar product on the right leads to (1.15).

Having shown that Theorem 1.7 implies 1.13 and 1.15 has prepared us to show that the same theorem also implies Corollary 1.9 .

Since the right-hand side of 1.16 is a symmetric polynomial in $x$ it may be expanded in terms of the Macdonald polynomials as in the left-hand side. The exercise is thus to determine the coefficients $c_{\lambda \mu}^{k}(q)$. Using 1.12 we obtain from (1.16) the identity

$$
\sum_{\lambda} c_{\lambda \mu}^{k}(q)\left\langle P_{\lambda}, P_{\nu}\right\rangle^{\prime}=\left\langle P_{\mu} h_{k-1, k}, P_{\nu}\right\rangle^{\prime}
$$

By the orthogonality 1.15 this yields

$$
c_{\lambda \mu}^{k}(q)=\frac{\left\langle P_{\mu} h_{k-1, k}, P_{\lambda}\right\rangle^{\prime}}{\left\langle P_{\lambda}, P_{\lambda}\right\rangle^{\prime}}
$$

where we have replaced $\nu$ by $\lambda$. Thanks to 1.13 and 1.15 , both scalar products can be explicitly computed resulting in the expression for $c_{\lambda \mu}^{k}(q)$ as claimed in (1.17).

Somewhat more general but easily derivable from Corollary 1.9 is the following $q$-binomial theorem.

Corollary 3.3. Let $\mu=\left(\mu_{1}, \ldots, \mu_{n}\right)$ be a partition and $a$ and $k$ integers such that $a \in\{0, \ldots, k-1\}$. Then

$$
\sum_{\lambda \in P} c_{\lambda \mu}^{a, k}(q) P_{\lambda}\left(x ; q, q^{k}\right)=P_{\mu}\left(x ; q, q^{k}\right) \prod_{i=1}^{n}\left(x_{i} ; q\right)_{a}\left(\frac{q}{x_{i}} ; q\right)_{k-a-1}
$$


where

$$
\begin{aligned}
c_{\lambda \mu}^{a, k}(q)=(-1)^{|\lambda|+|\mu|} & \left.\prod_{i=1}^{n} q^{\left(\lambda_{i}-\mu_{i}\right.}\right)\left[\begin{array}{c}
k-1 \\
a+\mu_{i}-\lambda_{i}
\end{array}\right]_{q} \\
& \times \prod_{1 \leq i<j \leq n} \frac{\left(q^{(j-i) k+\mu_{i}-\mu_{j}} ; q\right)_{k}\left(q^{1-k+(j-i) k+\lambda_{i}-\lambda_{j}} ; q\right)_{k}}{\left(q^{1+a-k+(j-i) k+\mu_{i}-\lambda_{j}} ; q\right)_{k}\left(q^{-a+(j-i) k+\lambda_{i}-\mu_{j}} ; q\right)_{k}} .
\end{aligned}
$$

Here we again adopt the convention of Theorem 1.7 that $c_{\lambda \mu}^{a, k}(q)=0$ unless $a+\mu_{i}-\lambda_{i} \in\{0, \ldots, k-1\}$ for all $i \in\{1, \ldots, n\}$.

Proof. Replace $\lambda \mapsto \lambda-(k-a-1)$ and $x \mapsto q^{k-a-1} x$. Using (2.5), 2.6) and (3.7) it then follows that we reduce to the case $a=k-1$.

3.6. Proof of Theorem 1.10. Key in our proof is the following sum over the structure constants of Macdonald polynomials.

Proposition 3.2. If $\lambda=\left(\lambda_{1}, \ldots, \lambda_{n}\right)$ and $\mu=\left(\mu_{1}, \ldots, \mu_{n}\right)$ are partitions then

$$
\sum_{\nu} f_{\mu \nu}^{\lambda} \frac{(q / t)_{\nu}}{c_{\nu}^{\prime}}=t^{(1-n)|\mu|} P_{\mu}\left(t^{\delta}\right) \frac{\left(q t^{n-1}\right)_{\lambda}}{c_{\lambda}^{\prime}} \prod_{i, j=1}^{n} \frac{\left(q t^{j-i-1} ; q\right)_{\lambda_{i}-\mu_{j}}}{\left(q t^{j-i} ; q\right)_{\lambda_{i}-\mu_{j}}} .
$$

Below we present two proofs of this result. The first and longer proof explains the origin of the above identity. The second and shorter proof uses Corollary 1.9 but obscures what is really going on. First, however, we show how 3.20 leads to Theorem 1.10 Multiplying both sides of $(3.20)$ by $P_{\lambda}(x)$ and summing over $\lambda$ yields

$$
\sum_{\lambda} \frac{\left(q t^{n-1}\right)_{\lambda} P_{\lambda}(x)}{c_{\lambda}^{\prime}} \prod_{i, j=1}^{n} \frac{\left(q t^{j-i-1} ; q\right)_{\lambda_{i}-\mu_{j}}}{\left(q t^{j-i} ; q\right)_{\lambda_{i}-\mu_{j}}}=t^{(n-1)|\mu|} \frac{P_{\mu}(x)}{P_{\mu}\left(t^{\delta}\right)}{ }_{1} \Phi_{0}(q / t ;-; q, t, x),
$$

where we have used $(3.9)$ and definition 2.16$)$. Summing the ${ }_{1} \Phi_{0}$ series by the $q$-binomial theorem (2.17) completes the proof.

First proof of Proposition 3.2. The proof hinges on the simple identity

$$
f_{\lambda \mu}^{\nu}=f_{\mu \hat{\nu}}^{\hat{\lambda}} \frac{\left\langle P_{\lambda}, P_{\lambda}\right\rangle^{\prime}}{\left\langle P_{\nu}, P_{\nu}\right\rangle^{\prime}}
$$

where, as before, the partitions $\hat{\lambda}$ and $\hat{\nu}$ are the complements of $\lambda$ and $\nu$ with respect to $\left(N^{n}\right)$, with $\nu_{1}$ and $\lambda_{1}$ not exceeding $N$. The identity (3.21) may seem to lack the necessary symmetry, but we note (see also the proof below) that $\left\langle P_{\lambda}, P_{\lambda}\right\rangle^{\prime}=$ $\left\langle P_{\hat{\lambda}}, P_{\hat{\lambda}}\right\rangle^{\prime}$.

The following proof of (3.21) is correct for all $t$ provided we take the more general definition of the scalar product 1.12 in terms of an $n$-dimensional integral as given in [20, Equation (VI.9.10)]. However, since (3.21) is a rational function identity it certainly suffices to establish its truth for just $t=q^{k}$. We leave it to the reader to either take $t=q^{k}$ in all of the remainder or to assume the generalized definition of the scalar product $\langle\cdot, \cdot\rangle^{\prime}$.

From the orthogonality of the Macdonald polynomials with respect to the scalar product $\langle\cdot, \cdot\rangle^{\prime}$ it follows that

$$
f_{\lambda \mu}^{\nu}=\frac{\left\langle P_{\lambda} P_{\mu}, P_{\nu}\right\rangle^{\prime}}{\left\langle P_{\nu}, P_{\nu}\right\rangle^{\prime}}
$$


Let $\bar{f}(x)=f\left(x_{1}^{-1}, \ldots, x_{n}^{-1}\right)$ and $e_{n}(x)=x_{1} \ldots x_{n}$. Then, by $\langle\cdot f, \cdot\rangle^{\prime}=\langle\cdot, \cdot \bar{f}\rangle^{\prime}$, $e_{n} \bar{e}_{n}=1$ and (3.14),

$$
f_{\mu \hat{\nu}}^{\hat{\lambda}}=\frac{\left\langle P_{\mu} P_{\hat{\nu}}, P_{\hat{\lambda}}\right\rangle^{\prime}}{\left\langle P_{\hat{\lambda}}, P_{\hat{\lambda}}\right\rangle^{\prime}}=\frac{\left\langle e_{n}^{N} P_{\mu} \bar{P}_{\nu}, e_{n}^{N} \bar{P}_{\lambda}\right\rangle^{\prime}}{\left\langle e_{n}^{N} \bar{P}_{\lambda}, e_{n}^{N} \bar{P}_{\lambda}\right\rangle^{\prime}}=\frac{\left\langle P_{\lambda} P_{\mu}, P_{\nu}\right\rangle^{\prime}}{\left\langle P_{\lambda}, P_{\lambda}\right\rangle^{\prime}}=f_{\lambda \mu}^{\nu} \frac{\left\langle P_{\nu}, P_{\nu}\right\rangle^{\prime}}{\left\langle P_{\lambda}, P_{\lambda}\right\rangle^{\prime}} .
$$

We now take $z=t^{n} q^{-N}$ in the identity of Proposition 3.1 and make the substitution (3.21). Also using [20, Equation (VI.9.6)]

$$
\frac{\left\langle P_{\lambda}, P_{\lambda}\right\rangle^{\prime}}{\left\langle P_{\nu}, P_{\nu}\right\rangle^{\prime}}=\frac{\left(q t^{n-1}\right)_{\nu}}{\left(q t^{n-1}\right)_{\lambda}} \frac{c_{\lambda}^{\prime}}{c_{\nu}^{\prime}} \frac{P_{\lambda}\left(t^{\delta}\right)}{P_{\nu}\left(t^{\delta}\right)}
$$

then gives

$$
\sum_{\nu} f_{\mu \hat{\nu}}^{\hat{\lambda}} \frac{\left(q t^{n-1}, q^{-N}\right)_{\nu}}{\left(q^{-N} t^{n}\right)_{\nu} c_{\nu}^{\prime}}=P_{\mu}\left(t^{\delta}\right) \frac{\left(q t^{n-1}\right)_{\lambda}}{c_{\lambda}^{\prime}} \prod_{i, j=1}^{n} \frac{\left(q^{-N} t^{n-i-j+1} ; q\right)_{\lambda_{i}+\mu_{j}}}{\left(q^{-N} t^{n-i-j+2} ; q\right)_{\lambda_{i}+\mu_{j}}} .
$$

Next we replace the summation index $\nu$ by $\hat{\nu}$ and eliminate $\hat{\nu}$ and $\lambda$ in favour of $\nu$ and $\hat{\lambda}$ using [6, Equation (4.1)]

$$
(a)_{\hat{\lambda}}=(-q / a)^{|\lambda|} t^{n(\lambda)} q^{n\left(\lambda^{\prime}\right)-N|\lambda|} \frac{(a)_{\left(N^{n}\right)}}{\left(q^{1-N} t^{n-1} / a\right)_{\lambda}}
$$

and [6, Equation (4.5)]

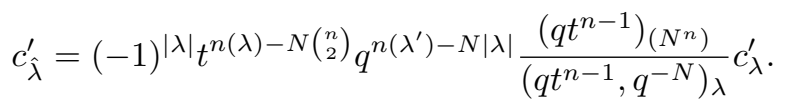

Therefore

$$
\sum_{\hat{\nu}} f_{\mu \nu}^{\hat{\lambda}} \frac{(q / t)_{\nu}}{c_{\nu}^{\prime}}=t^{(1-n)|\mu|} P_{\mu}\left(t^{\delta}\right) \frac{\left(q t^{n-1}\right)_{\hat{\lambda}}}{c_{\hat{\lambda}}^{\prime}} \prod_{i, j=1}^{n} \frac{\left(q t^{j-i-1} ; q\right)_{\hat{\lambda}_{i}-\mu_{j}}}{\left(q t^{j-i} ; q\right)_{\hat{\lambda}_{i}-\mu_{j}}}
$$

where we have also used that $f_{\mu \nu}^{\hat{\lambda}}=0$ unless $|\mu|+|\nu|=|\hat{\lambda}|$ and

$$
\frac{(a ; q)_{n-k}}{(b ; q)_{n-k}}=\frac{(a ; q)_{n}}{(b ; q)_{n}} \frac{\left(q^{1-n} / b ; q\right)_{k}}{\left(q^{1-n} / b ; q\right)_{k}}\left(\frac{b}{a}\right)^{k} .
$$

Next note that summing over the partition $\hat{\nu}$ is equivalent to summing over $\nu$. Indeed, $\left(N^{n}\right) \supset \hat{\nu}$ implies that also $\left(N^{n}\right) \supset \nu$. But since $f_{\mu \nu}^{\hat{\lambda}}=0$ if $\nu_{1}>\hat{\lambda}_{1}=N-\lambda_{1}$ we may simply sum over all partitions $\nu=\left(\nu_{1}, \ldots, \nu_{n}\right)$. Finally renaming $\hat{\lambda}$ as $\lambda$ completes the proof.

Second proof of Proposition 3.2. Since $f_{\mu \nu}^{\lambda}=0$ unless $|\lambda|=|\mu|+|\mu|$, only a finite number of terms contribute to the sum on the left. Hence for fixed $\lambda$ and $\mu$ equation (3.20) is a rational function identity. It thus suffices to give a proof for sufficiently many values of $t$, and in the following we will show that $\sqrt{3.20}$ is true for $t=q^{k}$ with $k$ a positive integer. 
We take 1.16 , replace $x$ by $q^{1-k} x$ and then expand the product on the right using (2.18). Hence

$$
\begin{aligned}
\operatorname{RHS} 1.16]\left.\right|_{x \mapsto x q^{1-k}} & =P_{\mu}\left(x q^{1-k} ; q, q^{k}\right)_{1} \Phi_{0}\left(q^{1-k} ;-; q, q^{k}, x\right) \\
& =q^{(1-k)|\mu|} P_{\mu}\left(x ; q, q^{k}\right) \sum_{\nu} \frac{\left(q^{1-k} ; q, q^{k}\right)_{\nu}}{c_{\nu}^{\prime}\left(q, q^{k}\right)} P_{\nu}\left(x ; q, q^{k}\right) \\
& =q^{(1-k)|\mu|} \sum_{\lambda, \nu} f_{\mu \nu}^{\lambda}\left(q, q^{k}\right) \frac{\left(q^{1-k} ; q, q^{k}\right)_{\nu}}{c_{\nu}^{\prime}\left(q, q^{k}\right)} P_{\lambda}\left(x ; q, q^{k}\right) .
\end{aligned}
$$

Equating coefficients of $P_{\lambda}\left(x ; q, q^{k}\right)$ with the left-hand side of 1.16$)$ (with $x \mapsto$ $x q^{1-k}$ ) yields

$$
\sum_{\nu} f_{\mu \nu}^{\lambda}\left(q, q^{k}\right) \frac{\left(q^{1-k} ; q, q^{k}\right)_{\nu}}{c_{\nu}^{\prime}\left(q, q^{k}\right)}=q^{(k-1)(|\mu|-|\lambda|)} c_{\lambda \mu}^{k}(q) .
$$

By (1.7), (3.1), (3.7) and 3.13 this is in accordance with equation (3.20) for $t=q^{k}$.

\section{BACK to Askey}

It seems appropriate to end this paper with another one of Askey's conjectures. By examining a special limit of the Askey-Habsieger-Kadell integral (Conjecture 1.3 with $\lambda=0$ ) Askey was led to conjecture the sum [3, Conjecture 7]

$$
\begin{array}{r}
\sum_{\lambda_{1}, \ldots, \lambda_{n}=0}^{N} \prod_{i=1}^{n}\left(\begin{array}{c}
\lambda_{i}+a-1 \\
\lambda_{i}
\end{array}\right)\left(\begin{array}{c}
N-\lambda_{i}+b-1 \\
N-\lambda_{i}
\end{array}\right) \prod_{1 \leq i<j \leq n}\left(1-k+\lambda_{j}-\lambda_{i}\right)_{2 k} \\
=\prod_{i=1}^{n} \frac{(a)_{(i-1) k}(b)_{(i-1) k}(a+b)_{N+(i-1) k}(i k) !}{(a+b)_{(n+i-2) k}(N-(i-1) k) ! k !} .
\end{array}
$$

This is contained in Askey's last $q$-integral [3, Conjecture 8] proved by Evans [7, Kadell 15 and Tarasov and Varchenko [25, Theorem E.8]. Although we are at present unable to use the Macdonald machinery to deal with Askey's last conjecture, we will show that the multivariable basic hypergeometric series studied in this paper can certainly deal with 4.1.

Let us begin by introducing the series

$$
\begin{aligned}
{ }_{r+1} \tilde{\Phi}_{r}\left[\begin{array}{c}
a_{1}, \ldots, a_{r+1} \\
b_{1}, \ldots, b_{r}
\end{array} ; q, z\right] & \\
& =\sum_{\lambda_{1}, \ldots, \lambda_{n}=0}^{\infty} \prod_{i=1}^{n} \frac{\left(a_{1}, \ldots, a_{r+1} ; q\right)_{\lambda_{i}} z^{\lambda_{i}}}{\left(q, b_{1}, \ldots, b_{r} ; q\right)_{\lambda_{i}}} \prod_{1 \leq i<j \leq n} q^{2 k \lambda_{i}}\left(q^{1-k+\lambda_{j}-\lambda_{i}} ; q\right)_{2 k}
\end{aligned}
$$

for $k$ a nonnegative integer and $n$ a positive integer. When $k=0$ this of course trivializes to the $n$-th power of an ordinary ${ }_{r+1} \phi_{r}$ series. We now claim the following $n$-dimensional version of the $q$-Pfaff-Saalschütz summation. 
Theorem 4.1. For $N$ a nonnegative integer there holds

$$
\begin{aligned}
{ }_{3} \tilde{\Phi}_{2}\left[\begin{array}{c}
a, b, q^{-N} \\
c, a b q^{1-N+2(n-1) k} / c
\end{array} ;, q\right] & \\
=q^{2 k^{2}\left(\begin{array}{c}
n \\
3
\end{array}\right)+k\left(\begin{array}{c}
n \\
2
\end{array}\right)} \prod_{i=1}^{n}\left(\frac{(q ; q)_{i k}}{(q ; q)_{k}}\right. & \frac{\left(a, b, q^{-N} ; q\right)_{(n-i) k}}{\left(c, a b q^{1-N+2(n-1) k} / c ; q\right)_{(n-i) k}} \\
& \left.\times \frac{\left(c q^{(1-i) k} / a, c q^{(1-i) k} / b ; q\right)_{N-(n-1) k}}{\left(c q^{(n-i) k}, c q^{(2-n-i) k} / a b ; q\right)_{N-(n-1) k}}\right) .
\end{aligned}
$$

Note that for $N<(n-1) k$ the right-hand side vanishes as it should. Indeed, because the summand on the left is zero for $\left|\lambda_{i}-\lambda_{j}\right| \in\{0, \ldots, k\}$ or $\max \left\{\lambda_{i}\right\}_{i=1}^{n}>$ $N$, there are no non-zero contributions to the sum when $N<(n-1) k$.

To see how the theorem relates to 4.1) we let $b$ tend to zero and then replace $a \mapsto q^{a}$ and $c \mapsto q^{1-N-b}$. By the usual manipulations involving $q$-shifted factorials, this leads to

$$
\begin{array}{r}
\sum_{\lambda_{1}, \ldots, \lambda_{n}=0}^{N} \prod_{i=1}^{n} q^{b \lambda_{i}}\left[\begin{array}{c}
\lambda_{i}+a-1 \\
\lambda_{i}
\end{array}\right]_{q}\left[\begin{array}{c}
N-\lambda_{i}+b-1 \\
N-\lambda_{i}
\end{array}\right]_{q} \prod_{1 \leq i<j \leq n} q^{2 k \lambda_{i}}\left(q^{1-k+\lambda_{j}-\lambda_{i}} ; q\right)_{2 k} \\
=q^{2 k^{2}\left(\begin{array}{c}
n \\
3
\end{array}\right)+b k\left(\begin{array}{c}
n \\
2
\end{array}\right)} \prod_{i=1}^{n} \frac{\left(q^{a}, q^{b} ; q\right)_{(i-1) k}\left(q^{a+b} ; q\right)_{N+(i-1) k}(q ; q)_{i k}}{\left(q^{a+b} ; q\right)_{(n+i-2) k}(q ; q)_{N-(i-1) k}(q ; q)_{k}}
\end{array}
$$

which is the obvious $q$-analogue of (4.1). For $k=1(4.2)$ is equivalent to [19, Theorem 6] of Krattenthaler.

Proof of Theorem 4.1. The theorem is really nothing but a rewriting of the PfaffSaalschütz sum for Macdonald polynomials due to Baker and Forrester [6, Equation $4.7]$

$$
{ }_{3} \Phi_{2}\left[\begin{array}{c}
a, b, q^{-N} \\
c, a b t^{n-1} q^{1-N} / c
\end{array} ; q, t, q t^{\delta}\right]=\frac{(c / a, b / a)_{\left(N^{n}\right)}}{(c, c / a b)_{\left(N^{n}\right)}} .
$$

Taking $t=q^{k}$ and using 3.3 this can be written as

$$
\begin{aligned}
& \sum_{\lambda} \frac{\left(a, b, q^{-N} ; q, q^{k}\right)_{\lambda} q^{2 k n(\lambda)+|\lambda|}}{\left(q^{1+(n-1) k}, c, a b q^{1-N+(n-1) k} / c ; q, q^{k}\right)_{\lambda}} \\
& \quad \times \prod_{1 \leq i<j \leq n}\left(1-q^{(j-i) k+\lambda_{i}-\lambda_{j}}\right)\left(q^{1+(j-i-1) k+\lambda_{i}-\lambda_{j}} ; q\right)_{2 k-1} \\
& \quad=\frac{\left(c / a, b / a ; q, q^{k}\right)_{\left(N^{n}\right)}}{\left(c, b / a b ; q, q^{k}\right)_{\left(N^{n}\right)}} \prod_{i=1}^{n} \frac{1-q^{k}}{1-q^{i k}} \frac{(q ; q)_{(i-1) k}(q ; q)_{i k}}{(q ; q)_{k}} .
\end{aligned}
$$

Multiplying both sides by

$$
\prod_{i=1}^{n} \frac{\left(a q^{(1-n) k}, b q^{(1-n) k}, q^{-N+(1-n) k} ; q\right)_{(n-i) k}}{\left(q, c q^{(1-n) k}, a b q^{1-N} / c ; q\right)_{(n-i) k}}
$$


and using 2.9$)$ and $(3.2)$, this becomes

$$
\begin{aligned}
\sum_{\lambda} & \prod_{i=1}^{n} \frac{\left(a q^{(1-n) k}, b q^{(1-n) k}, q^{-N+(1-n) k} ; q\right)_{\lambda_{i}+(n-i) k} q^{\lambda_{i}}}{\left(q, c q^{(1-n) k}, a b q^{1-N} / c ; q\right)_{\lambda_{i}+(n-i) k}} \\
& \times \prod_{1 \leq i<j \leq n} q^{2 k \lambda_{j}}\left(1-q^{(j-i) k+\lambda_{i}-\lambda_{j}}\right)\left(q^{1+(j-i-1) k+\lambda_{i}-\lambda_{j}} ; q\right)_{2 k-1} \\
= & \frac{\left(c / a, b / a ; q, q^{k}\right)_{\left(N^{n}\right)}}{\left(c, c / a b ; q, q^{k}\right)_{\left(N^{n}\right)}} \\
& \times \prod_{i=1}^{n} \frac{1-q^{k}}{1-q^{i k}} \frac{(q ; q)_{i k}}{(q ; q)_{k}} \frac{\left(a q^{(1-n) k}, b q^{(1-n) k}, q^{-N+(1-n) k} ; q\right)_{(n-i) k}}{\left(c q^{(1-n) k}, a b q^{1-N} / c ; q\right)_{(n-i) k}}
\end{aligned}
$$

Because 4.3 is zero for $N \in\{-(n-1) k, \ldots,-1\}$ the above sum is true for all integers $N$ such that $N+(n-1) k \geq 0$. Next we replace $a \mapsto a q^{(n-1) k}, b \mapsto b q^{(n-1) k}$, $c \mapsto c q^{(n-1) k}$ and $N \mapsto N-(n-1) k$. This yields

$$
\begin{aligned}
& \sum_{\lambda} \prod_{i=1}^{n} \frac{\left(a, b, q^{-N} ; q\right)_{\lambda_{i}+(n-i) k} q^{\lambda_{i}}}{\left(q, c, a b q^{1-N+2(n-1) k} / c ; q\right)_{\lambda_{i}+(n-i) k}} \\
& \times \prod_{1 \leq i<j \leq n} q^{2 k \lambda_{i}}\left(1-q^{(j-i) k+\lambda_{i}-\lambda_{j}}\right)\left(q^{1+(j-i-1) k+\lambda_{i}-\lambda_{j}} ; q\right)_{2 k-1} \\
&=\prod_{i=1}^{n}\left(\frac{1-q^{k}}{1-q^{i k}} \frac{(q ; q)_{i k}}{(q ; q)_{k}} \frac{\left(a, b, q^{-N} ; q\right)_{(n-i) k}}{\left(c, a b q^{1-N+2(n-1) k} / c ; q\right)_{(n-i) k}}\right. \\
&\left.\times \frac{\left(c q^{(1-i) k} / a, b q^{(1-i) k} / a ; q\right)_{N-(n-1) k}}{\left(c q^{(n-i) k}, c q^{(2-n-i) k} / a b ; q\right)_{N-(n-1) k}}\right)
\end{aligned}
$$

where $N$ should now be a nonnegative integer. Defining

$$
\begin{aligned}
h_{\lambda}=q^{-2 k^{2}\left(\begin{array}{c}
n \\
3
\end{array}\right)-k\left(\begin{array}{c}
n \\
2
\end{array}\right)} \prod_{i=1}^{n} \frac{\left(a, b, q^{-N} ; q\right)_{\lambda_{i}} q^{\lambda_{i}}}{\left(q, c, a b q^{1-N+2(n-1) k} / c ; q\right)_{\lambda_{i}}} \\
\times \prod_{1 \leq i<j \leq n} q^{2 k \lambda_{i}}\left(1-q^{\lambda_{i}-\lambda_{j}}\right)\left(q^{1-k+\lambda_{i}-\lambda_{j}} ; q\right)_{2 k-1}
\end{aligned}
$$

the left-hand side of (4.4) is nothing but $\sum_{\lambda} h_{\lambda+k \delta}$. Since $h_{\lambda}$ satisfies (3.5a) and $3.5 \mathrm{~b}$ we may utilize the unilateral version of Lemma 3.1 obtained by replacing the sum on the left of (3.6) by $\sum_{\lambda}$ and the sum on the right of 3.6 by $\sum_{\lambda_{1}, \ldots, \lambda_{n}=0}^{\infty}$. Provided we take $t=q^{k}$ in the unilateral form of (3.6) and use the symmetry of $h_{\lambda}$, we obtain Theorem 4.1 .

Acknowledgements. I thank Hjalmar Rosengren for helpful discussions.

\section{REFERENCES}

1. G. E. Andrews, R. Askey and R. Roy, Special functions, Encyclopedia of Mathematics and its Applications, Vol. 71, (Cambridge University Press, Cambridge, 1999).

2. K. Aomoto, Connection formulas of the q-analog de Rham cohomology, in Functional Analysis on the Eve of the 21st Century, Vol. 1, pp.1-12, S. Gindikin et al. eds., Prog. in Math. 131 (Birkhauser, Boston, MA, 1995).

3. R. Askey, Some basic hypergeometric extensions of integrals of Selberg and Andrews, SIAM J. Math. Anal. 11 (1980), 938-951. 
4. R. Askey, Ramanujan's extensions of the gamma and beta functions, Amer. Math. Monthly 87 (1980), 346-359.

5. R. Askey, Beta integrals in Ramanujan's papers, his unpublished work and further examples, in Ramanujan revisited, pp. 561-590, G. E. Andrews et al. eds., (Academic Press, Boston, MA, 1988).

6. T. H. Baker and P. J. Forrester, Transformation formulas for multivariable basic hypergeometric series, Methods Appl. Anal. 6 (1999), 147-164.

7. R. J. Evans, Multidimensional beta and gamma integrals, in The Rademacher legacy to mathematics, pp. 341-357, G. E. Andrews et al. eds., Contemp. Math. 166 (AMS, Providence, RI, 1994).

8. G. Gasper and M. Rahman, Basic Hypergeometric Series, Encyclopedia of Mathematics and its Applications, Vol. 35, (Cambridge University Press, Cambridge, 1990).

9. L. Habsieger, Une q-intégrale de Selberg et Askey, SIAM J. Math. Anal. 19 (1988), 1475-1489.

10. L. K. Hua, Harmonic analysis of functions of several complex variables in the classical domains, Translations of Mathematical Monographs, Vol. 6 (AMS, Providence, RI, 1979).

11. K. W. J. Kadell, A proof of some q-analogues of Selberg's integral for $k=1$, SIAM J. Math. Anal. 19 (1988), 944-968.

12. K. W. J. Kadell, A proof of Askey's conjectured q-analogue of Selberg's integral and a conjecture of Morris, SIAM J. Math. Anal. 19 (1988), 969-986.

13. K. W. J. Kadell, An integral for the product of two Selberg-Jack symmetric polynomials, Compositio Math. 87, (1993), 5-43.

14. K. W. J. Kadell, The Selberg-Jack symmetric functions, Adv. Math. 130 (1997), 33-102.

15. K. W. J. Kadell, A simple proof of an Aomoto-type extension of Askey's last conjectured Selberg q-integral, J. Math. Anal. Appl. 261 (2001), 419-440.

16. J. Kaneko, q-Selberg integrals and Macdonald polynomials, Ann. Sci. École Norm. Sup. (4) 29 (1996), 583-637.

17. J. Kaneko, Constant term identities of Forrester-Zeilberger-Cooper, Discrete Math. 173, (1997), 79-90.

18. J. Kaneko, $A_{1} \Psi_{1}$ summation theorem for Macdonald polynomials, Ramanujan J. 2 (1998), 379-386.

19. C. Krattenthaler, Schur function identities and the number of perfect matchings of holey Aztec rectangles, in q-Series from a Contemporary Perspective, pp. 335-349, M. E. H. Ismail and D. W. Stanton eds., Contemp. Math. 254 (AMS, Providence, RI, 2000).

20. I. G. Macdonald, Symmetric functions and Hall polynomials, second edition, (Oxford University Press, New-York, 1995).

21. I. G. Macdonald, Hypergeometric functions II, arXiv:1309.5208.

22. S. C. Milne, Summation theorems for basic hypergeometric series of Schur function argument, in Progress in approximation theory, pp. 51-77, A. A. Gonchar and E. B. Saff eds., Springer Ser. Comput. Math. 19 (Springer, New York, 1992).

23. W. G. Morris, Constant Term Identities for Finite Affine Root Systems: Conjectures and Theorems, Ph.D. dissertation, University of Wisconsin-Madison, 1982.

24. A. Selberg, Bemerkninger om et multipelt integral, Norske Mat. Tidsskr. 26 (1944), 71-78.

25. V. Tarasov and A. Varchenko, Geometry of q-hypergeometric functions, quantum affine algebras and elliptic quantum groups, Astérisque 246, (SMF, Paris, 1997).

26. Z. Yan, A class of generalized hypergeometric functions in several variables, Canad. J. Math. 44 (1992), 1317-1338.

Department of Mathematics and Statistics, The University of Melbourne, ViC 3010 , Australia

Email address: warnaar@ms.unimelb.edu.au 